\title{
Number-theoretic positive entropy shifts with small centralizer and large normalizer
}

\author{
M. BAAKE $\dagger$, Á. BUSTOS $\dagger$, C. HUCK $\dagger$, M. LEMAŃCZYK $\ddagger$ and A. NICKEL $\S$ \\ $\dagger$ Fakultät für Mathematik, Universität Bielefeld, Postfach 100131, \\ 33501 Bielefeld, Germany \\ (e-mail: mbaake,abustos,huck@math.uni-bielefeld.de) \\ $\ddagger$ Faculty of Mathematics and Computer Science, Nicolaus Copernicus University, \\ 12/18 Chopin Street, 87-100 Toruń, Poland \\ (e-mail:mlem@mat.umk.pl) \\ $\S$ Fakultät für Mathematik, Universität Duisburg-Essen, \\ Thea-Leymann-Str. 9, 45127 Essen, Germany \\ (e-mail: andreas.nickel@uni-due.de)
}

(Received 23 January 2020 and accepted in revised form 31 August 2020)

\begin{abstract}
Higher-dimensional binary shifts of number-theoretic origin with positive topological entropy are considered. We are particularly interested in analysing their symmetries and extended symmetries. They form groups, known as the topological centralizer and normalizer of the shift dynamical system, which are natural topological invariants. Here, our focus is on shift spaces with trivial centralizers, but large normalizers. In particular, we discuss several systems where the normalizer is an infinite extension of the centralizer, including the visible lattice points and the $k$-free integers in some real quadratic number fields.
\end{abstract}

Key words: number-theoretic shift spaces, topological centralizer and normalizer, $k$-free integers in number fields

2020 Mathematics Subject Classification: 37B10, 11H06 (Primary); 52C23, 28A80 (Secondary)

\section{Introduction}

Shift spaces under the action of $\mathbb{Z}^{d}$ form a much-studied class of dynamical systems, both for $d=1$ (cf. [28]) and for $d \geqslant 2$. In the latter case, much less is known in terms of general classifications, and even subclasses such as those of algebraic origin [34] are still rather enigmatic, despite displaying fascinating facets that have been intensively analysed. In particular, one is looking for interesting topological invariants to help analyse the jungle, and quite a bit of progress in this direction has been made recently. 
Among the available tools are the automorphism group of a shift space and its various siblings and generalizations; see [2,15-19] and references therein. Here, we adopt the point of view of $[2,9]$ to analyse both the (topological) centralizer (denoted by $\mathcal{S}$ below) and the normalizer of the shift space, the latter denoted by $\mathcal{R}$, as this pair can be quite revealing as soon as $d \geqslant 2$. In fact, both the topological setting and the extension to higher dimensions go beyond some of the initial studies $[21,26]$ that specifically looked at reversibility in the measure-theoretic setting for $d=1$; see $[2,31,33]$ and references therein for more on the early reversibility results. Further, the groups $\mathcal{S}$ and $\mathcal{R}$ are often explicitly accessible, both for systems of low complexity, where $\mathcal{S}$ is often minimal due to some form of topological rigidity, and beyond, where other rigidity mechanisms of a more algebraic nature emerge.

Below, we consider binary shift spaces of number-theoretic origin, as motivated by recent progress on $\mathcal{B}$-free systems and weak model sets; see [6, 19, 20, 23] and references therein. By way of characteristic examples with pure point spectrum, we demonstrate that positive topological entropy may very well be compatible with small or trivial centralizers, which means that $\mathcal{S}$ agrees with the underlying lattice (meaning a cocompact discrete subgroup of $\mathbb{R}^{d}$ ) or a finite-index extension thereof, but also that such systems may have considerably larger normalizers, which is of particular interest for $d \geqslant 2$. In fact, as shown in $[9,13]$, it is the group $\mathcal{R}$ that captures some obvious symmetries, as visible from the chair tiling and related shift spaces with their pertinent geometric symmetries. Also, the computability of $\mathcal{S}$ and $\mathcal{R}$ in these cases can be an advantage over some of the more general, abstract (semi-)groups that are presently attracting renewed attention.

The paper is organized as follows. After the introduction of some concepts and notions in $\S 2$, we set the scene with the well-known example of the visible lattice points of $\mathbb{Z}^{2}$ in $\S 3$, leading to Proposition 3.2 and Corollary 3.4, which in particular show that one has $\mathcal{R}=\mathbb{Z}^{2} \rtimes \operatorname{GL}(2, \mathbb{Z})$. Then $\S 4$ states and proves this for $\mathbb{Z}^{d}$ with $d \geqslant 2$ (Theorem 4.1) and introduces the general framework of lattice-based shift spaces, which can often be characterized by a rather powerful admissibility condition for its elements (Proposition 4.4). Then, under some mild assumptions, the normalizers are always maximal extensions of the corresponding centralizers (Theorem 4.5), with elements that are affine mappings (Corollary 4.7).

Section 5 explains the general number-theoretic setting of an algebraic $\mathcal{B}$-free system in higher dimensions, based on the classic Minkowski embedding of (commutative) maximal orders and their ideals as lattices in $\mathbb{R}^{d}$ for a suitable $d$. Here, Theorem 5.3 states the results on the triviality of $\mathcal{S}$ and the direct product nature of $\mathcal{R}$, which are true under a coprimality condition of the ideals chosen for $\mathcal{B}$ and a mild convergence condition, together known as the Erdôs property, in generalization of the one-dimensional notion [19] from $\mathcal{B}$-free integers.

Sections 6 and 7 then cover some paradigmatic examples from quadratic number fields. In the complex case, we treat the shift spaces generated by the $k$-free Gaussian or the $k$-free Eisenstein integers (Theorems 6.4 and 6.5). In both cases, $\mathcal{R}$ is the extension of $\mathcal{S} \simeq \mathbb{Z}^{2}$ by a maximal finite subgroup of $\operatorname{GL}(2, \mathbb{Z})$, which is substantially different from the case of the visible lattice points. Finally, in the real case, we consider $k$-free integers in the maximal order of $\mathbb{Q}(\sqrt{m})$ for $m \in\{2,3,5\}$. Here, Theorem 7.3 states that $\mathcal{R}$ is the semi-direct product of $\mathcal{S} \simeq \mathbb{Z}^{2}$ with a non-trivial infinite subgroup of $\operatorname{GL}(2, \mathbb{Z})$, which can 
be given a clear interpretation in terms of algebraic number theory. The latter case, which to the best of our knowledge is the first example of this type, is intermediate between known examples from inflation tilings and shifts such as that generated by the visible lattice points. Thus, it looks particularly promising for future work and extensions to general number fields.

\section{Preliminaries}

Let $\Gamma \subset \mathbb{R}^{d}$ be a lattice in $d$-space, that is, a discrete and cocompact subgroup of $\mathbb{R}^{d}$. Below, we will be working with the full shift (or configuration) space $\{0,1\}^{\Gamma}$, equipped with the standard product topology, and certain of its closed subspaces (called subshifts or simply shifts). We will generally use $\mathbb{X}$ to denote such a shift space, referring to either the full shift or the subshift under consideration. When the situation is independent of the geometry of the lattice, we will choose $\Gamma=\mathbb{Z}^{d}$ for simplicity. Any element $x \in \mathbb{X}$ can also be viewed as a subset of $\Gamma$, by taking the support of $x$, that is, by mapping $x$ to

$$
U_{x}:=\operatorname{supp}(x)=\left\{n \in \Gamma: x_{n}=1\right\} \subseteq \Gamma .
$$

Conversely, any point set $U \subseteq \Gamma$ can also be viewed as a configuration, by mapping it to $x_{U}=1_{U}$, that is, to its characteristic function. As usual, $\mathbb{X}$ admits a continuous action of $\Gamma$ on it, defined by $T: \Gamma \times \mathbb{X} \longrightarrow \mathbb{X}$, where $T(t, x)=T_{t}(x)$ with $\left(T_{t}(x)\right)_{n}:=x_{n+t}$. When working with $\mathbb{Z}^{d}$, we shall usually refer to its standard basis as $\left\{e_{1}, \ldots, e_{d}\right\}$, and align this with the elementary shift action of the $d$ commuting shift operators $T_{e_{i}}$. For the action of $\mathbb{Z}^{d}$ in this case, with $t=\left(t_{1}, \ldots, t_{d}\right)$, this simply means $T_{t}(x)=T_{e_{1}}^{t_{1}} \cdots T_{e_{d}}^{t_{d}}(x)$ for all $x \in\{0,1\}^{\mathbb{Z}^{d}}$.

Likewise, there is an action of $\Gamma$ on its subsets defined by

$$
\alpha_{t}(U)=t+U:=\{t+u: u \in U\} .
$$

It is easy to check that $U_{T_{t}(x)}=\alpha_{-t}\left(U_{x}\right)$. If we denote the power set of $\Gamma$ by $\Omega$, we thus get the commutative diagram

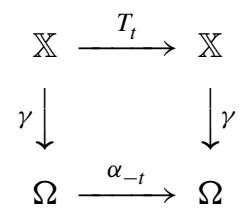

where $\gamma$ is the mapping defined by $x \mapsto U_{x}$. This is a homeomorphism if we equip $\Omega$ with the local topology, where two subsets of $\Gamma$ are $\varepsilon$-close to one another when they agree on the ball of radius $1 / \varepsilon$ around 0 . Consequently, by a slight abuse of notation, we will not distinguish these two points of view whenever the context is clear. This means that we will consider a subset $U \subseteq \Gamma$ simultaneously as a configuration, and vice versa.

In this spirit, we also consider the group of lattice automorphisms, $\operatorname{Aut}(\Gamma) \simeq \operatorname{GL}(d, \mathbb{Z})$. Indeed, if $\operatorname{Aut}(\mathbb{X})$ denotes the group of homeomorphisms of $\mathbb{X}$, any $M \in \operatorname{Aut}(\Gamma)$ induces an element $h_{M} \in \operatorname{Aut}(\mathbb{X})$, where

$$
\left(h_{M}(x)\right)_{n}:=x_{M^{-1} n} \cdot
$$


In fact, the mapping $M \mapsto h_{M}$ defines an injective group homomorphism. Here, one can check that $U_{h_{M}(x)}=M U_{x}$, so the counterpart to (2.1) is the commutative diagram

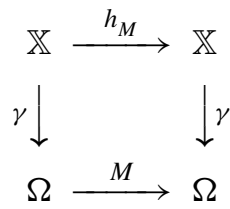

which makes calculations with elements of the form $h_{M}$ more convenient in the formulation with subsets. From now on, we identify $\mathbb{X}$ and $\Omega$, and use the symbol $\mathbb{X}$ for both. For ease of understanding, we will normally use $x, y$ for configurations and $U, V$ for sets.

A point set $S \subset \mathbb{R}^{d}$, by which we mean an at most countable union of singleton sets, is said to have natural density if

$$
\operatorname{dens}(S)=\lim _{r \rightarrow \infty} \frac{\operatorname{card}\left(S \cap B_{r}\right)}{\operatorname{vol}\left(B_{r}\right)}
$$

exists, where $B_{r}$ denotes the closed ball of radius $r$ around 0 . One can use other sets for averaging, as long as they are centred around 0 and satisfy some condition of Følner or van Hove type; see $[4,8]$ for details.

Below, we shall need the following simple result on sublattices of a given lattice, where the term sublattice is meant to include the property that the corresponding index is finite.

FACT 2.1. Let $\Gamma$ be a lattice in $\mathbb{R}^{d}$, and let $\Gamma_{1}$ and $\Gamma_{2}$ be sublattices of $\Gamma$, with corresponding indices $m_{1}$ and $m_{2}$, respectively. Then $\Gamma_{1} \cap \Gamma_{2}$ and $\Gamma_{1}+\Gamma_{2}$ are sublattices of $\Gamma$ as well.

Further, if the indices $m_{1}$ and $m_{2}$ are coprime, one has $\Gamma_{1}+\Gamma_{2}=\Gamma$, which implies that $\Gamma_{1}$ meets all cosets of $\Gamma_{2}$ and vice versa.

Proof. If $\left[\Gamma: \Gamma_{i}\right]=m_{i}$, one has $m_{i} \Gamma \subseteq \Gamma_{i}$ by standard arguments, which implies

$$
m_{1} m_{2} \Gamma \subseteq \Gamma_{1} \cap \Gamma_{2} \subseteq \Gamma_{1}+\Gamma_{2} \subseteq \Gamma .
$$

The sublattice property for $\Gamma_{1} \cap \Gamma_{2}$ and $\Gamma_{1}+\Gamma_{2}$ is then clear.

The next statement is a consequence of what is sometimes referred to as the diamond isomorphism theorem, but can also be seen directly as follows. Set $n=\left[\Gamma:\left(\Gamma_{1}+\Gamma_{2}\right)\right]$ and $n_{i}=\left[\left(\Gamma_{1}+\Gamma_{2}\right): \Gamma_{i}\right]$. Then, for $i \in\{1,2\}$,

$$
m_{i}=\left[\Gamma: \Gamma_{i}\right]=\left[\Gamma:\left(\Gamma_{1}+\Gamma_{2}\right)\right]\left[\left(\Gamma_{1}+\Gamma_{2}\right): \Gamma_{i}\right]=n n_{i},
$$

which implies $n \mid \operatorname{gcd}\left(m_{1}, m_{2}\right)=1$ and thus $\Gamma_{1}+\Gamma_{2}=\Gamma$. The final implication for the cosets is a now simple consequence.

An important concept for shift spaces is that of a block map; see [28] for background. When working with subshifts $\mathbb{X} \subseteq \mathcal{A}^{\mathbb{Z}^{d}}$ and $\mathbb{Y} \subseteq \mathcal{B}^{\mathbb{Z}^{d}}$ over finite alphabets $\mathcal{A}$ and $\mathcal{B}$, a continuous mapping $h: \mathbb{X} \longrightarrow \mathbb{Y}$ is called a block map if there is a non-negative integer $\ell$ such that, for every $x \in \mathbb{X}$ and all $n \in \mathbb{Z}^{d}$, the image $y=h(x)$ at position $n$ is fully determined from the patch $\left\{x_{n+m}: m \in[-\ell, \ell]^{d}\right\}$, that is, from the knowledge of $x$ within a $d$-cube of sidelength $2 \ell$ centred at $n$. In other words, the action of $h$ can be seen as 
the result of a sliding block code $\phi=\phi_{h}$ that, for some fixed $\ell \in \mathbb{N}_{0}$, maps a cubic block of $(2 \ell+1)^{d}$ symbols from $\mathcal{A}$ to a single letter from $\mathcal{B}$, positioned at the centre of the block (which can easily be modified when needed). This is the symbolic version of a local derivation rule from discrete geometry $[4, \S 5.2]$. An important result that we shall need repeatedly is the Curtis-Hedlund-Lyndon (CHL) theorem: if a continuous mapping $h: \mathbb{X} \longrightarrow \mathbb{Y}$ intertwines the shift action on $\mathbb{X}$ and $\mathbb{Y}$, it must be a block map based on some code $\phi$ of the above type [28, Theorem 6.2.9].

Below, we shall only be interested in subshifts on which the action of $\Gamma$ is faithful, which means that the subshift contains non-periodic elements. In this context, it is also natural to consider the affine lattice group $\Gamma \rtimes \operatorname{Aut}(\Gamma)$, whose elements $(t, M)$ act on $\mathbb{R}^{d}$ via $(t, M)(y):=M y+t$, and correspondingly on $\mathbb{X}$. In this formulation, the group multiplication is $(t, M)(s, N)=(t+M s, M N)$, with neutral element $(0, \mathbb{1})$ and inverse elements $(t, M)^{-1}=\left(-M^{-1} t, M^{-1}\right)$. This group will become important later.

Further notation and concepts can now better be introduced with a paradigmatic example, which will simultaneously motivate the various extensions to follow.

\section{Visible lattice points and their shift space}

Consider the visible points $V$ of the square lattice, $\mathbb{Z}^{2}$, which are defined as

$$
V:=\left\{(m, n) \in \mathbb{Z}^{2}: \operatorname{gcd}(m, n)=1\right\} .
$$

They are also known as the primitive points, and are used in many places; see also the cover page of [1]. Clearly, one has $V=\mathbb{Z}^{2} \backslash \bigcup_{p \in \mathcal{P}}\left(p \mathbb{Z}^{2}\right)$, where $\mathcal{P}$ denotes the set of rational primes. Figure 1 shows a finite patch around the origin, in comparison with another set that will be discussed later, in $\S 6$. Let us recall some well-known properties of $V$; see $[4,8]$ and references therein for background and further results.

FACT 3.1. The set $V$ is uniformly discrete, but not relatively dense. In particular, $V$ contains holes of unbounded inradius that repeat lattice-periodically. Yet, it satisfies $V-V=\mathbb{Z}^{2}$ and has natural density dens $(V)=1 / \zeta(2)=6 / \pi^{2}$, where $\zeta(s)$ is Riemann's zeta function.

Furthermore, the set $V$ is pure point diffractive, with the diffraction measure being invariant under the action of the affine group $\mathbb{Z}^{2} \rtimes \mathrm{GL}(2, \mathbb{Z})$.

Now, let $\mathbb{X}_{V}:=\overline{\mathbb{Z}^{2}+V}$ be the orbit closure of $V$ under the shift (or translation) action of $\mathbb{Z}^{2}$, where the closure is taken in the standard product topology, also known as the local topology due to its geometric interpretation: two configurations (or subsets) are close if they agree on a large neighbourhood of $0 \in \mathbb{Z}^{2}$. In particular, since $V$ has holes of arbitrary size, one immediately obtains that $\varnothing \in \mathbb{X}_{V}$, where $\varnothing$ is the empty set and represents the all-0 configuration. Clearly, $\mathbb{X}_{V}$ is a compact space, which is canonically identified with a subshift in $\{0,1\}^{\mathbb{Z}^{2}}$, and $\left(\mathbb{X}_{V}, \mathbb{Z}^{2}\right)$ is a topological dynamical system.

Call a subset of $\mathbb{Z}^{2}$ admissible if it misses at least one coset modulo $p \mathbb{Z}^{2}$ for any $p \in \mathcal{P}$. One easily verifies that the set of admissible sets constitutes a subshift of $\{0,1\}^{\mathbb{Z}^{2}}$ as well, denoted by $\mathbb{A}$. Since the set $V$ by the remark above misses the zero coset modulo each $p \mathbb{Z}^{2}$, one readily verifies that the elements of $\mathbb{X}_{V}$ are admissible, so $\mathbb{X}_{V} \subseteq \mathbb{A}$. In fact, it 


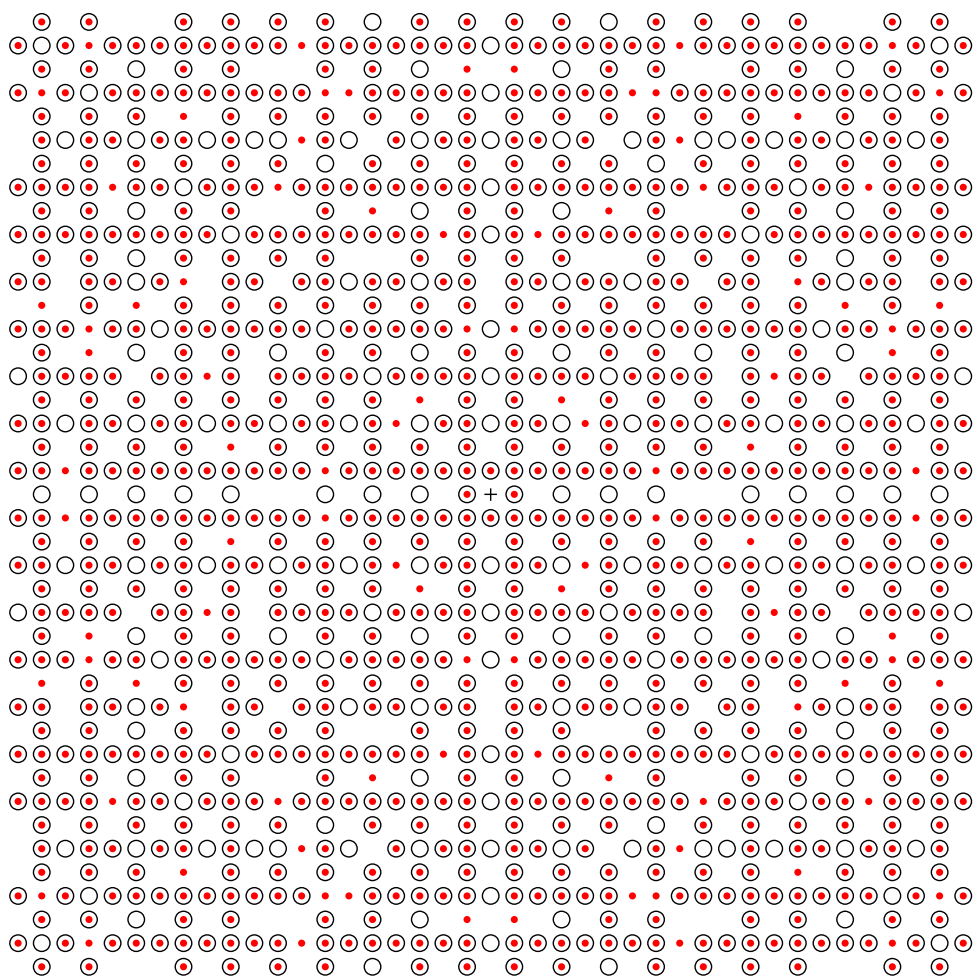

FIGURE 1. Central patch of the visible points of $\mathbb{Z}^{2}$ (dots) and of the square-free Gaussian integers (circles). The cross in the centre marks the origin

was shown in [5, Lemma 4] that $V$ shows all cosets except the zero coset modulo each $p \mathbb{Z}^{2}$, and is thus a maximal element of $\mathbb{X}_{V}$. Further, one has the following result, the first part of which will be generalized below on the basis of Propositions 4.4 and 5.2.

Proposition 3.2 [5]. The space $\mathbb{X}_{V}$ coincides with the shift space of admissible sets, $\mathbb{A}$. In particular, $\mathbb{X}_{V}$ is hereditary (closed under the formation of subsets). The topological dynamical system $\left(\mathbb{X}_{V}, \mathbb{Z}^{2}\right)$ has topological entropy $\left(6 / \pi^{2}\right) \log (2)$.

With respect to the existing natural frequency measure $v_{\mathrm{M}}$, which is also known as the Mirsky measure, the measure-theoretic dynamical system $\left(\mathbb{X}_{V}, \mathbb{Z}^{2}, v_{\mathrm{M}}\right)$ has pure point dynamical spectrum, but trivial topological point spectrum.

The measure $v_{M}$ is ergodic for the $\mathbb{Z}^{2}$-action, and $V$ is a generic element for $v_{M}$ in $\mathbb{X}_{V}$. Moreover, the measure-theoretic entropy for $v_{\mathrm{M}}$ vanishes.

The characterization of a number-theoretic shift space via an admissibility condition was originally observed by Sarnak for the square-free integers, and later extended to Erdős $\mathcal{B}$-free numbers in [20] and generalized to the lattice setting in [32]. Since this step is vital to us, we later present a streamlined version of the proof that covers the generality we need.

Remark 3.3. The generating shifts induce unitary operators on the Hilbert space $L^{2}\left(\mathbb{X}_{V}, v_{M}\right)$, and the simultaneous eigenfunctions form a basis of this space [6, 7]. Except 
for the trivial one, no other eigenfunction is continuous. However, as follows from a recent result by Keller [24] (see also the discussion in $[6,10]$ ), there is a subset of $\mathbb{X}_{V}$ of full measure on which the eigenfunctions are continuous. This is related to the fact that $V$ is a weak model set of maximal density [6] in the cut and project scheme $\left(\mathbb{R}^{2}, H, \mathcal{L}\right)$ with compact internal group $H=\prod_{p \in \mathcal{P}} \mathbb{Z}^{2} / p \mathbb{Z}^{2}$ and the lattice $\mathcal{L}$ being the diagonal embedding of $\mathbb{Z}^{2}$ into $\mathbb{R}^{2} \times H$. It is an interesting open problem to understand the missing null set, and to connect it with the rather intricate relation between the topological and the measure-theoretic structure of this dynamical system.

Let $\operatorname{Aut}\left(\mathbb{X}_{V}\right)$ be the automorphism group of $\mathbb{X}_{V}$, by which we mean the group of all homeomorphisms of $\mathbb{X}_{V}$, irrespective of whether they commute with the generators $T_{1}, T_{2}$ of the $\mathbb{Z}^{2}$-action or not. The translation action of $\mathbb{Z}^{2}$ on $\mathbb{X}_{V}$ is faithful, as a result of which we have $\mathcal{G}:=\left\langle T_{1}, T_{2}\right\rangle \simeq \mathbb{Z}^{2}$. Clearly, $\mathbb{X}_{V}$ is not the full shift, and $\varnothing$ is the only fixed point of $\mathbb{X}_{V}$ under the translation action, since $\mathbb{Z}^{2}$ (as the all-1 configuration) is not an element of $\mathbb{X}_{V}$.

The symmetry group of $\mathbb{X}_{V}$ (see [2] and references therein for background) is

$$
\mathcal{S}\left(\mathbb{X}_{V}\right):=\operatorname{cent}_{\operatorname{Aut}\left(\mathbb{X}_{V}\right)}(\mathcal{G})=\left\{H \in \operatorname{Aut}\left(\mathbb{X}_{V}\right): G H=H G \text { for all } G \in \mathcal{G}\right\},
$$

which clearly contains $\mathcal{G}$ as a normal subgroup. This centralizer is often called the automorphism group of the subshift, denoted by $\operatorname{Aut}\left(\mathbb{X}_{V}, \mathcal{G}\right)$, but we prefer to avoid the potential confusion with the automorphism group in the above (or Smale) sense. For any $S \in \mathcal{S}\left(\mathbb{X}_{V}\right), S(V)$ has a dense shift orbit in $\mathbb{X}_{V}$ (as $V$ has dense orbit by definition). Moreover, one has $S(\varnothing)=\varnothing$ since $S(\varnothing)$ can also be seen as a fixed point under the translation action.

Now, consider an arbitrary $S \in \mathcal{S}\left(\mathbb{X}_{V}\right)$. By the CHL theorem, there is a block code (or map) $\phi:\{0,1\}^{[-\ell, \ell]^{2}} \longrightarrow\{0,1\}$ of a suitable size (parameterized by $\ell$ ) such that, for any $x \in \mathbb{X}_{V}$, the value of $S x$ at a position $k \in \mathbb{Z}^{2}$ is given by the value under $\phi$ of the corresponding block of $x$ around this very position, which we call its centre. This means

$$
(S x)_{k}=\phi\left(x_{\left[k+[-\ell, \ell]^{2}\right]}\right),
$$

where $x_{\left[k+[-\ell, \ell]^{2}\right]}(m)=x_{k+m}$ for $m \in[-\ell, \ell]^{2}$. Since $S(\varnothing)=\varnothing$, it is clear that $\phi\left(0_{[-\ell, \ell]^{2}}\right)=0$.

Next, following $[2,9]$, we define the extended symmetry group as

$$
\mathcal{R}\left(\mathbb{X}_{V}\right):=\operatorname{norm}_{\operatorname{Aut}\left(\mathbb{X}_{V}\right)}(\mathcal{G})=\left\{H \in \operatorname{Aut}\left(\mathbb{X}_{V}\right): H \mathcal{G} H^{-1}=\mathcal{G}\right\},
$$

which contains both $\mathcal{G}$ and $\mathcal{S}\left(\mathbb{X}_{V}\right)$ as normal subgroups. Every $H \in \mathcal{R}\left(\mathbb{X}_{V}\right)$ must satisfy $H(\varnothing)=\varnothing$, as $H(\varnothing)$ can once again be shown to be fixed under any element of $\mathcal{G}$. Since every (extended) symmetry induces an automorphism of $\mathcal{G} \simeq \mathbb{Z}^{2}$ via the conjugation action, $\mathcal{R}\left(\mathbb{X}_{V}\right)$ can at most be a group extension of $\mathcal{S}\left(\mathbb{X}_{V}\right)$ by $\operatorname{Aut}\left(\mathbb{Z}^{2}\right)=\mathrm{GL}(2, \mathbb{Z})$.

Let us state the final result for this specific example, which is a special case of our more general statement (Theorem 4.1) in the next section. 
COROLLARY 3.4. For the topological dynamical system $\left(\mathbb{X}_{V}, \mathbb{Z}^{2}\right)$, the symmetry group is the minimal one, so $\mathcal{S}\left(\mathbb{X}_{V}\right)=\mathcal{G} \simeq \mathbb{Z}^{2}$, while the extended symmetry group is

$$
\mathcal{R}\left(\mathbb{X}_{V}\right)=\mathcal{S}\left(\mathbb{X}_{V}\right) \rtimes \operatorname{Aut}\left(\mathbb{Z}^{2}\right) \simeq \mathbb{Z}^{2} \rtimes \mathrm{GL}(2, \mathbb{Z}),
$$

hence the maximal extension possible.

This result shows that positive (topological) entropy is highly compatible with a minimal centralizer, while the factor group $\mathcal{R}\left(\mathbb{X}_{V}\right) / \mathcal{S}\left(\mathbb{X}_{V}\right)$ need neither be a finite nor a periodic group, where the latter statement implies that the factor group contains elements of infinite order. This combination can also occur for subshifts with zero entropy, as can be seen from the subshift that is obtained as the orbit closure of a singleton configuration and contains the shift orbit of this configuration together with the all-0 configuration (cf. [4, Example 4.3]).

\section{General lattice setting}

The statement of Corollary 3.4 is not restricted to $d=2$. Indeed, one has the following generalization; see $[5,8,32]$ for its first part.

THEOREM 4.1. Let $V=\left\{\left(n_{1}, \ldots, n_{d}\right) \in \mathbb{Z}^{d}: \operatorname{gcd}\left(n_{1}, \ldots, n_{d}\right)=1\right\}=\mathbb{Z}^{d} \backslash \bigcup_{p}\left(p \mathbb{Z}^{d}\right)$ be the set of visible points of $\mathbb{Z}^{d}$, with $d \geqslant 2$, and consider the topological dynamical system $\left(\mathbb{X}_{V}, \mathbb{Z}^{d}\right)$ with $\mathbb{X}_{V}=\overline{\mathbb{Z}^{d}+V}$. Then $\mathbb{X}_{V}$ has topological entropy $\log (2) / \zeta(d)$ and satisfies $\mathbb{X}_{V}=\mathbb{A}$, where $\mathbb{A}$ consists of all admissible subsets of $\mathbb{Z}^{d}$, that is, all subsets $U \subset \mathbb{Z}^{d}$ such that, for every $p \in \mathcal{P}, U$ misses at least one coset modulo $p \mathbb{Z}^{d}$.

The symmetry group, or topological centralizer, of this system is $\mathcal{S}\left(\mathbb{X}_{V}\right)=\mathbb{Z}^{d}$, while its extended symmetry group, or topological normalizer, is $\mathcal{R}\left(\mathbb{X}_{V}\right)=\mathbb{Z}^{d} \rtimes \mathrm{GL}(d, \mathbb{Z})$.

Proof. The statement on the centralizer is a rigidity result that is driven by the identity $\mathbb{X}_{V}=\mathbb{A}$, which also forces $\mathbb{X}_{V}$ to be hereditary. It follows from a slight modification of the argument put forward in [29], which we repeat here in a form that is tailored to the higher-dimensional lattice systems we consider here and below. It employs a lattice version of the Chinese remainder theorem (CRT) based on the pairwise coprime sublattices of the form $p \mathbb{Z}^{d}$ ( $p$ prime) of the integer lattice. Note that the solutions of a system of congruences appear lattice-periodically, which guarantees some flexibility regarding the actual position of solutions in the square lattice. This argument also works for general lattices.

We start from the identity $\mathbb{X}_{V}=\mathbb{A}$, which follows from Proposition 3.2 together with its generalization in Proposition 4.4 and Theorem 4.5 below. First, we show that any symmetry $S \in \mathcal{S}\left(\mathbb{X}_{V}\right)$ acts on the singleton set $U_{0}=\{0\} \in \mathbb{X}_{V}$ as a translation, so $S\left(U_{0}\right)=U_{0}+k$ for some $k \in \mathbb{Z}^{d}$, where $U_{0} \in \mathbb{X}_{V}$ follows from $\mathbb{X}_{V}=\mathbb{A}$. Since $S$ is a homeomorphism that commutes with the shift action, it corresponds to a block code $\phi$, by the CHL theorem. Here and in what follows, we identify any subset of $\mathbb{Z}^{d}$ with its characteristic function, and thus with a binary configuration, as explained in $\S 2$. Then $S\left(U_{0}\right)=U_{0}+k$ is equivalent to saying that $\phi$ takes the value 1 on exactly one block with singleton support. For the latter, 
note first that $\phi$ cannot take the value 0 on all blocks with singleton support, as this would imply $S\left(U_{0}\right)=\varnothing$ which is impossible ( $S$ is invertible and we already have $S(\varnothing)=\varnothing$ ).

Assuming the existence of two different blocks with singleton support that are sent to 1 by the code, there exist a prime $p$ and an admissible set $U \subset \mathbb{Z}^{d}$ of cardinality $p^{d}-1$ that comprises all cosets modulo $p \mathbb{Z}^{d}$ except the zero coset, together with the property that $S(U)$ shows all possible cosets modulo this very $p \mathbb{Z}^{d}$ and is thus no longer admissible. To see this, $p$ is chosen such that the difference $n$ of the centres of the two blocks (a non-zero element of $\mathbb{Z}^{d}$ ) does not belong to $p \mathbb{Z}^{d}$. In fact, by the CRT, the $p^{d}-1$ elements of $U$ can be chosen arbitrarily well separated from one another. Then the assertion follows because $S(U)$ will contain a translate of $U \cup(n+U)$ and, since $S(U)$ is admissible, a translate of this union is contained in $V$. Thus, for some $m \in \mathbb{Z}^{d}$, both $U+m$ and $(U+n)+m$ consist of $p^{d}-1$ elements and are equal modulo $p \mathbb{Z}^{d}$ (both showing all non-zero cosets modulo $\left.p \mathbb{Z}^{d}\right)$ - a contradiction to $n \neq 0$ modulo $p \mathbb{Z}^{d}$ from the construction.

After replacing $S$ by $S^{\prime}:=T_{k} \circ S$, so that $S^{\prime}\left(U_{0}\right)=U_{0}$, and slightly enlarging the size of the block code, one can assume that the only block with singleton support that is sent to 1 is the block that has value 1 only at 0 . One is then left to show that $S^{\prime}=\mathrm{id}$. For convenience, we now rename $S^{\prime}$ as $S$, and show that $S=$ id.

This follows from the maximality of $V$ together with the crucial observation that $S(U) \subseteq U$ (equivalently $U \subseteq S^{-1}(U)$ ) for all $U \in \mathbb{X}_{V}$, due to the properties of the block code for $S$ just established. So, any (automatically admissible) block of $1_{U}$ with value 0 at its central position is sent to 0 by the code. This claim can be shown by an argument similar to the one used above. Assume the existence of an admissible block $C$ with value 0 at its centre that is sent to 1 by the code. This block then appears in $V$ at a position $s$ with $s \in p \mathbb{Z}^{d}$ for a suitable $p$. Again, one can choose a set $U$ of $p^{d}-1$ elements of $V$ that shows all cosets except the zero coset modulo $p \mathbb{Z}^{d}$. By the CRT, we may assume that these $p^{d}-1$ elements are well separated and also well separated from $s$ (together with the whole block $s+C$ of $V$ at $s)$. It is then immediate that $U \cup(s+C)$ is admissible and that $S(U \cup(s+C))$ will contain the set $U \cup\{s\}$ and thus shows all cosets modulo $p \mathbb{Z}^{d}$, a contradiction.

It remains to determine the normalizer. Since $\mathcal{G} \simeq \mathbb{Z}^{d}$, with $\operatorname{Aut}\left(\mathbb{Z}^{d}\right)=\operatorname{GL}(d, \mathbb{Z})$, there is a group homomorphism

$$
\psi: \mathcal{R}\left(\mathbb{X}_{V}\right) \longrightarrow \operatorname{GL}(d, \mathbb{Z})
$$

that is induced as follows. If $H \in \mathcal{R}\left(\mathbb{X}_{V}\right)$, we have $H \mathcal{G} H^{-1}=\mathcal{G}$, so a set of generators of $\mathcal{G}$ must be mapped to a (possibly different) set of generators under the conjugation action. Starting from our canonical choice, $\mathcal{G}=\left\langle T_{e_{1}}, \ldots, T_{e_{d}}\right\rangle$, one finds $H T_{i} H^{-1}=$ $\prod_{j} T_{j}^{m_{j i}}$ where the $m_{j i}$ are the matrix elements of $M_{H}=\psi(H)$. It is routine to verify the homomorphism property. In particular, with $T_{n}=T_{e_{1}}^{n_{1}} \cdots T_{e_{d}}^{n_{d}}$, one gets

$$
H T_{n} H^{-1}=T_{M_{H} n} .
$$

For $M \in \mathrm{GL}(d, \mathbb{Z})$, in line with equation (2.2), define the mapping $H_{M}$ on $\mathbb{X}=\{0,1\}^{\mathbb{Z}^{d}}$ by

$$
\left(H_{M} x\right)_{n}=x_{M^{-1} n},
$$


which clearly is a homeomorphism of $\mathbb{X}$. Now, each $M$ maps our set $V$ onto itself, as $\mathrm{GL}(d, \mathbb{Z})$ acts transitively on $V$. Consequently, the orbit $\left\{t+V: t \in \mathbb{Z}^{d}\right\}$ is also mapped onto itself by $M$, hence $M$ preserves $\mathbb{X}_{V}$ by continuity. In other words, invoking (2.3), we see that $H_{M}$ is an element of $\mathcal{R}\left(\mathbb{X}_{V}\right)$, and that

$$
1 \longrightarrow \mathcal{S}\left(\mathbb{X}_{V}\right) \stackrel{\text { id }}{\longrightarrow} \mathcal{R}\left(\mathbb{X}_{V}\right) \stackrel{\psi}{\longrightarrow} \mathrm{GL}(d, \mathbb{Z}) \longrightarrow 1
$$

is a short exact sequence. Moreover, the mapping

$$
\varphi: \operatorname{GL}(d, \mathbb{Z}) \longrightarrow \operatorname{Aut}\left(\mathbb{X}_{V}\right)
$$

defined by $\varphi(M)=H_{M}$ is a group homomorphism as well, with $\psi\left(H_{M}\right)=M$. Consequently, $\mathcal{H}:=\varphi(\mathrm{GL}(d, \mathbb{Z}))$ is a subgroup of $\mathcal{R}\left(\mathbb{X}_{V}\right)$ that is isomorphic with $\operatorname{GL}(d, \mathbb{Z})$. Since $\varphi \circ \psi$ acts as the identity on $\mathcal{H}$, our claim follows.

Remark 4.2. With respect to the patch frequency (or Mirsky) measure, the situation is also the same as for $d=2$, meaning that the dynamical spectrum of $\left(\mathbb{X}_{V}, \mathbb{Z}^{d}, v_{\mathrm{M}}\right)$ is pure point, with trivial topological point spectrum. Nevertheless, the measure-theoretic eigenfunctions are continuous on a subset of $\mathbb{X}_{V}$ of full measure; see the discussion in [6].

In fact, the above multi-dimensional setting allows for a further generalization.

Definition 4.3. Let $\mathcal{B}=\left\{b_{i} \mid i \in \mathbb{N}\right\}$ be an infinite set of positive integers that is primitive in the sense that $b_{i} \mid b_{j}$ implies $i=j$. Consider the point set $V_{\mathcal{B}}=\mathbb{Z}^{d} \backslash \bigcup_{i \in \mathbb{N}} b_{i} \mathbb{Z}^{d}$ in $\mathbb{R}^{d}$, and define $\mathbb{X}_{\mathcal{B}}=\overline{\mathbb{Z}^{d}+V_{\mathcal{B}}}$, which is compact. Then the dynamical system $\left(\mathbb{X}_{\mathcal{B}}, \mathbb{Z}^{d}\right)$ is called a $\mathcal{B}$-free lattice system. It is called Erdós when the $b_{i}$ are pairwise coprime and satisfy

$$
\sum_{i=0}^{\infty} \frac{1}{b_{i}^{d}}<\infty,
$$

which is an additional condition only for $d=1$.

Note that $d=1$ is the case of $\mathcal{B}$-free systems in $\mathbb{Z}$, which is extensively studied in $[19,23]$ and references therein. The primitivity condition really is an irreducibility notion, as any multiple of some $b_{i}$ could simply be removed from the set $\mathcal{B}$ without any effect on $V_{\mathcal{B}}$. It is obvious that $\mathbb{Z}^{d}$ in Definition 4.3 can be replaced by any lattice $\Gamma \subset \mathbb{R}^{d}$. However, since this does not change the arithmetic situation at hand, we restrict our attention to $\mathbb{Z}^{d}$ for now.

A set $U \subset \mathbb{Z}^{d}$ is called admissible for $\mathcal{B}$ if, for every $b \in \mathcal{B}, U$ meets at most $b^{d}-1$ cosets of the sublattice $b \mathbb{Z}^{d}$. Equivalently, $U$ is admissible if it misses at least one coset of $b \mathbb{Z}^{d}$ for each $b \in \mathcal{B}$. The set of all admissible subsets of $\mathbb{Z}^{d}$ is again denoted by $\mathbb{A}$, and constitutes a subshift. By definition, $V_{\mathcal{B}} \in \mathbb{A}$, and we thus have $\mathbb{X}_{\mathcal{B}} \subseteq \mathbb{A}$. If $P$ and $Q$ are disjoint finite subsets of $\mathbb{Z}^{d}$, we define the locator set

$$
L(P, Q):=\left\{t \in \mathbb{Z}^{d}: t+P \subset V_{\mathcal{B}} \text { and } t+Q \subset \mathbb{Z}^{d} \backslash V_{\mathcal{B}}\right\}
$$

by analogy with the treatment in [32]. One has the following connection, which is a generalization of both [20, Proposition 2.5] and [32, Theorem 2]. 
Proposition 4.4. Assume that $\left(\mathbb{X}_{\mathcal{B}}, \mathbb{Z}^{d}\right)$ is Erdós, and let $P$ and $Q$ be disjoint finite subsets of $\mathbb{Z}^{d}$. Then the following properties are equivalent.

(1) $L(P, Q)$ has positive natural density.

(2) $L(P, Q) \neq \varnothing$.

(3) $P$ is admissible for $\mathcal{B}$.

Proof. The implication (1) $\Rightarrow$ (2) is clear. If $L(P, Q) \neq \varnothing$, one has $t+P \subset V_{\mathcal{B}}$ for some $t \in \mathbb{Z}^{d}$, so $t+P \in \mathbb{A}$ and hence $P \in \mathbb{A}$, which shows (2) $\Rightarrow$ (3).

It remains to prove $(3) \Rightarrow(1)$. To this end, let $m=\operatorname{card}(P)$ and set

$$
S_{1}:=\left\{b \in \mathcal{B}: \min \left(\operatorname{card}(P \bmod b), b^{d}-1\right)<m\right\},
$$

which is a finite subset of $\mathcal{B}$. Further, for the elements $q \in Q$, select distinct elements $b_{q}$ from $\mathcal{B} \backslash S_{1}$, and set $S_{2}=\left\{b_{q}: q \in Q\right\}$. Without loss of generality, we may choose each $b_{q}$ large enough so that $p \equiv q \bmod b_{q}$ has no solution with $p \in P$, which is to say that $q$ is a representative of a coset modulo $b_{q}$ that is missed by $P$. Since $\operatorname{card}\left(S_{2}\right)=\operatorname{card}(Q)$, $S:=S_{1} \cup S_{2}$ is still a finite subset of $\mathcal{B}$, with $S=S_{1}$ for $Q=\varnothing$.

Since $P$ is admissible for $\mathcal{B}$, we know that, for each $b \in \mathcal{B}$, at least one coset of $b \mathbb{Z}^{d}$ is missed by $P$. Let $p_{b}$ be a representative of this coset, where we may choose $p_{b}=q$ for all $b=b_{q} \in S_{2}$ due to our choice of $S_{2}$. As our system is Erdős, we can invoke the lattice version of the CRT to see that there is an element $t_{0} \in \mathbb{Z}^{d}$ such that

$$
t_{0} \equiv-p_{b} \bmod b \quad \text { for all } b \in S \text {. }
$$

Note that, with the choice of the $p_{b}$ for $b \in S_{2}$ just made, this comprises the congruences $t_{0} \equiv-q \bmod b_{q}$ for all $q \in Q$. In fact, due to the pairwise coprimality, we know that the set of all solutions is given by the lattice coset $t_{0}+c \mathbb{Z}^{d}$ with $c=\prod_{b \in S} b$. For any $t$ from this coset and then every $b \in S$, we thus have $t+p \not \equiv 0 \bmod b$, which is to say that $t+P$ avoids the zero coset for all $b \in S$, while $t+q \equiv 0 \bmod b_{q}$, so no element of $t+Q$ can lie in $V_{\mathcal{B}}$.

Now, let $R_{n}:=\{b \in \mathcal{B} \backslash S: b \leqslant n\}$, which is finite, where we assume the integer $n$ to be large enough so that $R_{n} \neq \varnothing$. Consider

$$
\Theta_{n}:=\left(t_{0}+c \mathbb{Z}^{d}\right) \cap\left\{t \in \mathbb{Z}^{d}: t \not \equiv-p \bmod b \text { for all } b \in R_{n} \text { and all } p \in P\right\} .
$$

The second set is a finite union of translates of the lattice $\gamma_{n} \mathbb{Z}^{d}$ with $\gamma_{n}=\prod_{b \in R_{n}} b$. Invoking Fact 2.1, it is clear that $\Theta_{n}$ consists of finitely many cosets of the intersection lattice, which is $c \gamma_{n} \mathbb{Z}^{d}$, and thus has a well-defined natural density. Consequently, $\Theta_{n}$ has density

$$
\operatorname{dens}\left(\Theta_{n}\right)=c^{-d} \prod_{b \in R_{n}}\left(1-\frac{\operatorname{card}(P)}{b^{d}}\right)
$$

because, modulo $b$ for any $b \in R_{n}$, no two points of $P$ can be equal by our choice of $S_{1}$.

Each term in the product is a positive number, again due to our choice of $S_{1} \subseteq S$, so the Erdôs condition guarantees that the infinite product satisfies

$$
\prod_{b \in \mathcal{B} \backslash S}\left(1-\frac{\operatorname{card}(P)}{b^{d}}\right)=D>0,
$$


which is to say that it converges to a positive number. Since $\Theta_{n+1} \subseteq \Theta_{n}$ for all large enough $n$, say $n \geqslant n_{0}$, we can take the limit $n \rightarrow \infty$ and conclude that $\Theta_{\infty}:=\bigcap_{n \geqslant n_{0}} \Theta_{n}$ is a set of solutions of our congruence conditions, for all $b \in \mathcal{B}$, with positive natural density. So, for any $t \in \Theta_{\infty}$, we have $t+P \subset V_{\mathcal{B}}$ together with $t+Q \subset \mathbb{Z}^{d} \backslash V_{\mathcal{B}}$ as claimed.

THEOREM 4.5. Let $\left(\mathbb{X}_{\mathcal{B}}, \mathbb{Z}^{d}\right)$ be a $\mathcal{B}$-free lattice system, with symmetry group $\mathcal{S}=\mathcal{S}\left(\mathbb{X}_{\mathcal{B}}\right)$. Then the group of extended symmetries is given by $\mathcal{R}=\mathcal{R}\left(\mathbb{X}_{\mathcal{B}}\right)=\mathcal{S} \rtimes \operatorname{GL}(d, \mathbb{Z})$, which is to say that the extension is always the maximally possible one.

If $\left(\mathbb{X}_{\mathcal{B}}, \mathbb{Z}^{d}\right)$ is Erdôs, one has $\mathbb{X}_{\mathcal{B}}=\mathbb{A}$, the system is hereditary, and it has minimal symmetry group, $\mathcal{S}=\mathcal{G} \simeq \mathbb{Z}^{d}$, and we thus get $\mathcal{R}=\mathbb{Z}^{d} \rtimes \mathrm{GL}(d, \mathbb{Z})$.

Proof. Due to the assumptions, any $\mathcal{B}$-free lattice system defines a shift, with faithful shift action, so its symmetry group, $\mathcal{S}\left(\mathbb{X}_{\mathcal{B}}\right)$, contains a normal subgroup that is isomorphic with $\mathbb{Z}^{d}$, namely the one generated by the shift action itself, $\mathcal{G}$.

Since $\operatorname{Aut}\left(\mathbb{Z}^{d}\right)=\operatorname{GL}(d, \mathbb{Z})$, any $M \in \operatorname{GL}(d, \mathbb{Z})$ maps $\mathbb{Z}^{d}$ onto itself, hence one also has $M\left(b \mathbb{Z}^{d}\right)=b M\left(\mathbb{Z}^{d}\right)=b \mathbb{Z}^{d}$ for any $b \in \mathcal{B}$. This implies $M\left(V_{\mathcal{B}}\right)=V_{\mathcal{B}}$. We thus see that $\mathcal{H}:=\varphi(\mathrm{GL}(d, \mathbb{Z}))$ is a subgroup of $\mathcal{R}\left(\mathbb{X}_{\mathcal{B}}\right)$ that is isomorphic to $\operatorname{GL}(d, \mathbb{Z})$. Since we have $\psi(\mathcal{H})=\psi\left(\mathcal{R}\left(\mathbb{X}_{\mathcal{B}}\right)\right)=\operatorname{GL}(d, \mathbb{Z})$, where $\psi$ is the group homomorphism from above, $\mathcal{S}$ is the kernel of the group endomorphism $\varphi \circ \psi$. By construction, $\varphi \circ \psi$ acts as the identity on $\mathcal{H}$, and the claimed semi-direct product structure follows.

Clearly, we have $\mathbb{X}_{\mathcal{B}} \subseteq \mathbb{A}$, as explained earlier. For the converse inclusion, when $\mathbb{X}_{\mathcal{B}}$ is Erdős, consider an arbitrary $S \in \mathbb{A}$ and, for $n \in \mathbb{N}$, set $S_{n}=S \cap B_{n}(0)$, which is finite. By Proposition 4.4, for each $n \in \mathbb{N}$, there exists some $t_{n} \in L\left(S_{n},\left(\mathbb{Z}^{d} \cap B_{n}(0)\right) \backslash S_{n}\right) \neq \varnothing$, which means that

$$
\left(V_{\mathcal{B}}-t_{n}\right) \cap B_{n}(0)=S_{n} .
$$

Consequently, $\lim _{n \rightarrow \infty}\left(V_{\mathcal{B}}-t_{n}\right)=S$ in the local topology, and $S \in \mathbb{X}_{\mathcal{B}}$. This shows $\mathbb{A} \subseteq \mathbb{X}_{\mathcal{B}}$ and hence $\mathbb{X}_{\mathcal{B}}=\mathbb{A}$. Clearly, $\mathbb{X}_{\mathcal{B}}$ is then also hereditary. Now, a straightforward modification of the centralizer argument used in the proof of Theorem 4.1 establishes $\mathcal{S}=\mathbb{Z}^{d}$.

Alternatively, the structure of the last proof can be summarized by stating that

$$
1 \longrightarrow \mathcal{S} \stackrel{\text { id }}{\longrightarrow} \mathcal{R} \stackrel{\psi}{\longrightarrow} \mathrm{GL}(d, \mathbb{Z}) \longrightarrow 1
$$

is a short exact sequence where $\mathcal{H}:=\varphi(\mathrm{GL}(d, \mathbb{Z}))$ is a subgroup of $\mathcal{R}$ with $\mathcal{H} \simeq \mathrm{GL}(d, \mathbb{Z})$ and the property that $\varphi \circ \psi$ acts as the identity on $\mathcal{H}$. Outside the class of Erdős $\mathcal{B}$-free lattice systems, the centralizer can indeed be a finite-index extension of $\mathcal{G}$, as is known from one-dimensional examples of Toeplitz type [25], but we do not consider this case below.

Example 4.6. Let $k \in \mathbb{N}$ be fixed and consider the lattice $\mathbb{Z}^{d}$. Then $\mathcal{B}=\left\{p^{k}: p \in \mathcal{P}\right\}$ leads to the $k$-free lattice points in $d$ dimensions, which is Erdós for $k d \geqslant 2$. They have been studied from various angles in $[5,8,32]$, and provide a natural extension of our motivating example from $\S 3$. 
In particular, one always obtains a measure-theoretic dynamical system $\left(\mathbb{X}_{V_{\mathcal{B}}}, \mathbb{Z}^{d}, v_{\mathrm{M}}\right)$ with pure point diffraction and dynamical spectrum, as in Remark 4.2. The topological entropy is $\log (2) / \zeta(k d)$, while the measure-theoretic entropy with respect to the natural patch frequency (or Mirsky) measure $v_{M}$ always vanishes [32], as it must in view of the fact that the dynamical spectrum of $\left(\mathbb{X}_{V_{\mathcal{B}}}, \mathbb{Z}^{d}, v_{\mathrm{M}}\right)$ is pure point.

The result of Theorem 4.5 can be examined more generally as follows. Let $\left(\mathbb{X}, \mathbb{Z}^{d}\right)$ be a faithful shift, with centralizer $\mathcal{S}(\mathbb{X})$ and normalizer $\mathcal{R}(\mathbb{X})$, and assume that $h_{M} \in \operatorname{Aut}(\mathbb{X})$ for some $M \in \mathrm{GL}(d, \mathbb{Z})$, where $h_{M}$ is the mapping defined in equation (2.2). Let $T_{n}$ with $n \in \mathbb{Z}^{d}$ denote the shift by $n$ as before, so $\left(T_{n} x\right)_{m}=x_{m+n}$, and consider any $H \in \mathcal{R}(\mathbb{X})$ with $M=\psi(H)$. Then, for any $\ell \in \mathbb{Z}^{d}$, one obtains the commutative diagram

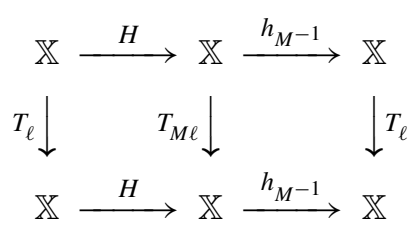

from equation (4.1), where $h_{M^{-1}} \in \operatorname{Aut}(\mathbb{X})$ by assumption. So, $h_{M^{-1}} \circ H \in \operatorname{Aut}(\mathbb{X})$ commutes with the shift action, hence is a block map by the CHL theorem.

At this point, the structure of the centralizer enters crucially, and one obtains an interesting consequence as follows, where $\psi: \mathcal{R}(\mathbb{X}) \longrightarrow \operatorname{Aut}\left(\mathbb{Z}^{d}\right)$ is the homomorphism from above.

COROLlary 4.7. Let $\left(\mathbb{X}, \mathbb{Z}^{d}\right)$ be a faithful subshift with trivial centralizer. Consider an element $H \in \mathcal{R}(\mathbb{X})$ with $h_{\psi(H)} \in \operatorname{Aut}(\mathbb{X})$. Then $H$ is an affine mapping and $h_{\psi(H)} \in \mathcal{R}(\mathbb{X})$.

Proof. From diagram (4.2), with $M=\psi(H)$, we know that $h_{M^{-1}} \circ H \in \mathcal{S}(\mathbb{X})$, so this mapping equals $T_{n}$ for some $n \in \mathbb{Z}^{d}$. This means $H=h_{M} \circ T_{n}$, which acts as

$$
(H x)_{m}=x_{M^{-1} m+n} .
$$

The equivalent formulation with sets, due to the relation $h_{M} \circ T_{n}=T_{M n} \circ h_{M}$, now reads $H(U)=-M n+M(U)$, which is affine.

Finally, since $H \in \mathcal{R}(\mathbb{X})$, one also has $h_{M}=H \circ T_{-n} \in \mathcal{R}(\mathbb{X})$.

The occurrence of affine mappings in the context of $\mathbb{Z}^{d}$-actions, as a sign of some degree of rigidity, is also known from [27, Theorem 1.1], and will become important later.

\section{Number-theoretic setting}

The concept of a $\mathcal{B}$-free lattice system from Definition 4.3 is only one possibility to generalize the one-dimensional notion. For another, combining methods from the theory of aperiodic order [4] with classic results from elementary and algebraic number theory $[12,30]$, one may start with the treatment of square-free integers in algebraic number fields as in [14], and simplify and generalize it as follows. 
Let $\mathbb{K}$ be an algebraic number field of degree $d$, so $[\mathbb{K}: \mathbb{Q}]=d<\infty$. Let $\mathcal{O}$ be the ring of integers in $\mathbb{K}$, which is the unique maximal order in $\mathbb{K}$, such as $\mathbb{Z}$ for $\mathbb{K}=\mathbb{Q}, \mathbb{Z}[i]$ for $\mathbb{K}=\mathbb{Q}(\mathrm{i})$, or $\mathbb{Z}[\sqrt{2}]$ for $\mathbb{K}=\mathbb{Q}(\sqrt{2})$. Let $\iota: \mathcal{O} \longrightarrow \mathbb{R}^{r} \times \mathbb{C}^{s}$ be the mapping defined by

$$
z \mapsto\left(\rho_{1}(z), \ldots, \rho_{r}(z), \sigma_{1}(z), \ldots, \sigma_{s}(z)\right),
$$

where $\rho_{1}, \ldots, \rho_{r}$ are the real embeddings of $\mathbb{K}$ into $\mathbb{C}$, while $\sigma_{1}, \ldots, \sigma_{s}$ arise from the complex embeddings of $\mathbb{K}$ into $\mathbb{C}$ by choosing exactly one embedding from each pair of complex conjugate ones (in particular, we have $d=r+2 s$ ). Clearly, depending on $\mathbb{K}$, one either takes $\rho_{1}$ or $\sigma_{1}$ to be the identity.

Now, if $\mathfrak{b}$ is a non-zero ideal of $\mathcal{O}$, its absolute norm is defined by $\mathrm{N}(\mathfrak{b}):=[\mathcal{O}: \mathfrak{b}]$. In fact, for any of the above choices, the image $\iota(\mathfrak{b})$ is a lattice in $\mathbb{R}^{r} \times \mathbb{C}^{s} \simeq \mathbb{R}^{d}$, and the absolute norm of $\mathfrak{b}$ is precisely the index of the sublattice $\iota(\mathfrak{b})$ in the lattice $\iota(\mathcal{O})$, and thus a finite number. The map $\iota$ is usually called the Minkowski embedding of $\mathcal{O}$; see $[4,12,30]$ for details.

To continue, let $\mathbb{K}$ be an algebraic number field of degree $d$, and $\mathcal{O}$ its ring of integers, with Minkowski embedding $\Gamma=\iota(\mathcal{O}) \subset \mathbb{R}^{d}$. Let $\mathcal{B}=\left\{\mathfrak{b}_{i} \mid i \in \mathbb{N}\right\}$ be an infinite set of non-trivial ideals of $\mathcal{O}$, where $\mathcal{B}$ is assumed to be primitive in the sense that $\mathfrak{b}_{i} \supseteq \mathfrak{b}_{j}$ implies $i=j$. Let $\Gamma_{i}=\iota\left(\mathfrak{b}_{i}\right)$ and consider $V_{\mathcal{B}}:=\Gamma \backslash \bigcup_{i \in \mathbb{N}} \Gamma_{i} \subset \mathbb{R}^{d}$, which thus is the Minkowski embedding of $\mathcal{O} \backslash \bigcup_{i \in \mathbb{N}} \mathfrak{b}_{i}$, and define its hull as the orbit closure

$$
\mathbb{X}_{\mathcal{B}}=\overline{\Gamma+V_{\mathcal{B}}}
$$

in the local topology, so $\mathbb{X}_{\mathcal{B}}$ is compact as in our previous examples.

Definition 5.1. In the setting just explained, the topological dynamical system $\left(\mathbb{X}_{\mathcal{B}}, \Gamma\right)$ is called an algebraic $\mathcal{B}$-free lattice system, or simply an algebraic $\mathcal{B}$-free system.

Such a system is called Erdös when the $\mathfrak{b}_{i}$ are pairwise coprime (meaning $\mathfrak{b}_{i}+\mathfrak{b}_{j}=\mathcal{O}$ for all $i \neq j$ ) and satisfy

$$
\sum_{i=1}^{\infty} \frac{1}{\mathrm{~N}\left(\mathfrak{b}_{i}\right)}<\infty
$$

As before, we call a set $U \subset \Gamma$ admissible for $\mathcal{B}$ when, for every $\mathfrak{b} \in \mathcal{B}$, the set $U$ meets at most $\mathrm{N}(\mathfrak{b})-1$ cosets of $\Gamma_{\mathfrak{b}}:=\iota(\mathfrak{b})$ in $\Gamma$, that is, misses at least one. All admissible subsets of $\Gamma$ once again constitute a subshift, denoted by $\mathbb{A}$, which contains $\mathbb{X}_{\mathcal{B}}$ by construction.

Proposition 5.2. Assume that $\left(\mathbb{X}_{\mathcal{B}}, \Gamma\right)$ is Erdös, and let $P$ and $Q$ be disjoint finite subsets of $\Gamma$. Then the following properties are equivalent.

(1) The locator set $L(P, Q):=\left\{t \in \Gamma: t+P \subset V_{\mathcal{B}}\right.$ and $\left.t+Q \subset \Gamma \backslash V_{\mathcal{B}}\right\}$ has positive natural density.

(2) $L(P, Q) \neq \varnothing$.

(3) $P$ is admissible for $\mathcal{B}$.

Proof. This is a variant of the proof of Proposition 4.4, where (1) $\Rightarrow(2) \Rightarrow(3)$ is again clear. We thus need to establish $(3) \Rightarrow(1)$. 
Let $m=\operatorname{card}(P)$ and choose $S_{1}$ as the set of all ideals $\mathfrak{b} \in \mathcal{B}$ with the properly that card $\left(P \bmod \Gamma_{\mathfrak{b}}\right)<m$ or $\mathrm{N}(\mathfrak{b}) \leqslant m$. As before, $S_{1}$ is finite. Then, for $S_{2}$, select distinct ideals from $\mathcal{B} \backslash S_{1}$, denoted by $S_{2}=\left\{\mathfrak{b}_{q}: q \in Q\right\}$, where, without loss of generality, we may select ideals $\mathfrak{b}_{q}$ of sufficiently large absolute norm such that $P$ does not meet the coset modulo $\mathfrak{b}_{q}$ represented by $q$. Then consider $S=S_{1} \cup S_{2}$, which is still finite. As all ideals $\mathfrak{b} \in \mathcal{B}$ can be viewed as lattices $\Gamma_{\mathfrak{b}}$ via the Minkowski embedding, we can again invoke the CRT to find an element $t_{0} \in \Gamma$ so that

$$
t_{0} \equiv-p_{\mathfrak{b}} \bmod \Gamma_{\mathfrak{b}} \quad \text { for all } \mathfrak{b} \in S
$$

where $p_{\mathfrak{b}}$ is a representative of a coset modulo $\Gamma_{\mathfrak{b}}$ that is missing in $P$, which we know to exist. Due to our construction of $S_{2}$, we may choose $p_{\mathfrak{b}_{q}}=q$ for all $q \in Q$, as a result of which the above congruences actually comprise $t_{0} \equiv-q \bmod \Gamma_{\mathfrak{b}_{q}}$ for all $q \in Q$. By pairwise coprimality of the $\mathfrak{b} \in \mathcal{B}$, we see that the set of all solutions is the coset $t_{0}+G$, where $G$ is the Minkowski embedding of the ideal $\prod_{\mathfrak{b} \in S} \mathfrak{b}$. For any $t$ from this coset, $t+P$ avoids the zero coset of $\Gamma_{\mathfrak{b}}$ for all $\mathfrak{b} \in S$, while no element of $t+Q$ is in $V_{\mathcal{B}}$, so $t+Q \subset \Gamma \backslash V_{\mathcal{B}}$.

Now, for $n \in \mathbb{N}$, consider the set $R_{n}:=\{\mathfrak{b} \in \mathcal{B} \backslash S: \mathrm{N}(\mathfrak{b}) \leqslant n\}$. For a suitable $n_{0}$ and then all $n \geqslant n_{0}$, the set $R_{n}$ is non-empty and finite. Next, define

$$
\Theta_{n}:=\left(t_{0}+G\right) \cap\left\{t \not \equiv-p \bmod \Gamma_{\mathfrak{b}} \text { for all } \mathfrak{b} \in R_{n} \text { and all } p \in P\right\}
$$

Then $\Theta_{n}$ is once again a finite union of translates of a non-trivial intersection lattice and thus a set of positive natural density, the latter being given by

$$
\operatorname{dens}(G) \prod_{\mathfrak{b} \in R_{n}}\left(1-\frac{\operatorname{card}(P)}{\mathrm{N}(\mathfrak{b})}\right)
$$

As in the previous case, the product is convergent as $n \rightarrow \infty$ by the Erdôs condition, so $\Theta_{\infty}:=\bigcap_{n \geqslant n_{0}} \Theta_{n}$ is a subset of $\Gamma$ of positive density such that, for any $t \in \Theta_{\infty}$, we have $t+P \subset V_{\mathcal{B}}$ and $t+Q \subset \Gamma \backslash V_{\mathcal{B}}$.

THEOREM 5.3. An Erdös algebraic $\mathcal{B}$-free system $\left(\mathbb{X}_{\mathcal{B}}, \Gamma\right)$ satisfies $\mathbb{X}_{\mathcal{B}}=\mathbb{A}$ and is hereditary. Moreover, it has minimal symmetry group, so $\mathcal{S}=\mathcal{S}\left(\mathbb{X}_{\mathcal{B}}\right)=\Gamma \simeq \mathbb{Z}^{d}$. Moreover, its extended symmetry group is of the form $\mathcal{R}\left(\mathbb{X}_{\mathcal{B}}\right)=\mathcal{S} \rtimes \mathcal{H}$, where $\mathcal{H}$ is isomorphic to a non-trivial subgroup of $\mathrm{GL}(d, \mathbb{Z})$.

Proof. While $\mathbb{X}_{\mathcal{B}} \subseteq \mathbb{A}$ is clear, $\mathbb{A} \subseteq \mathbb{X}_{\mathcal{B}}$ is shown exactly as in Theorem 4.5, this time on the basis of Proposition 5.2, so $\mathbb{X}_{\mathcal{B}}=\mathbb{A}$, and this shift is hereditary. Then the statement on the centralizer follows, once again, from a straightforward modification of the argument used in the proof of Theorem 4.1.

Let $\mathbb{X}=\mathbb{X}_{\mathcal{B}}$, and take an arbitrary $H \in \mathcal{R}(\mathbb{X})$. Here, we have $M:=\psi(H) \in \operatorname{Aut}(\Gamma)$ by analogy with our previous cases, and diagram (4.2) changes to 


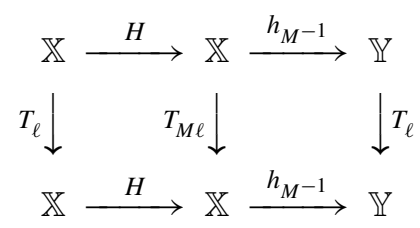

where $\mathbb{Y}:=h_{M^{-1}}(\mathbb{X})$, while $T_{\ell}$ with $\ell \in \Gamma$ is the shift in this case. Note that both $\mathbb{X}$ and $\mathbb{Y}$ are subshifts of $\{0,1\}^{\Gamma}$, on which $T_{n}$ and $h_{M}$ are still well defined, and it is clear that $\varnothing \in \mathbb{X} \cap \mathbb{Y}$. This new diagram is again commutative, so $\chi=h_{M^{-1}} \circ H$ intertwines the shift actions on $\mathbb{X}$ and $\mathbb{Y}$. Consequently, by the CHL theorem, $\chi$ is a block map.

The space $\mathbb{Y}$ inherits important properties from $\mathbb{X}$, such as its characterization through admissibility (now defined via the images of cosets in $\mathbb{X}$ under $h_{M^{-1}}$ ) as well as being hereditary. After minor modifications, the arguments from the proof of Theorem 4.1 now show that $\chi$ must be a shift map, hence equal to $T_{n}$ for some $n \in \Gamma$. But $T_{n} \mathbb{X}=\mathbb{X}$, whence $H \in \operatorname{Aut}(\mathbb{X})$ now implies

$$
\mathbb{Y}=h_{M^{-1}}(\mathbb{X})=h_{M^{-1}}(H \mathbb{X})=T_{n} \mathbb{X}=\mathbb{X}
$$

and we are back in the situation of Corollary 4.7. Consequently, $H$ is an affine mapping, with $H=h_{M} \circ T_{n}$, and $h_{M} \in \mathcal{R}(\mathbb{X})$. We thus have a short exact sequence

$$
1 \longrightarrow \mathcal{S}(\mathbb{X}) \stackrel{\text { id }}{\longrightarrow} \mathcal{R}(\mathbb{X}) \stackrel{\psi}{\longrightarrow} \mathcal{H}:=\psi(\mathcal{R}(\mathbb{X})) \longrightarrow 1
$$

with $\mathcal{H}$ a subgroup of $\operatorname{Aut}(\Gamma)$. In particular, we get $\mathcal{R}(\mathbb{X})=\mathcal{S}(\mathbb{X}) \rtimes \mathcal{H}$ as claimed.

To see that $\mathcal{H}$ is non-trivial, we observe that the unit group $\mathcal{O}^{\times}$is non-trivial (it contains at least the elements \pm 1 ) and, via the Minkowski embedding, isomorphic to a subgroup of $\operatorname{Aut}(\Gamma) \simeq \operatorname{GL}(d, \mathbb{Z})$. Each element of $\mathcal{O}^{\times}$maps any ideal $\mathfrak{b}$ onto itself, so the corresponding mapping induced by the Minkowski embedding is a bijection of $V_{\mathcal{B}}$, and thus gives rise to an extended symmetry. Further elements emerge from non-trivial Galois automorphisms of $\mathbb{K}$, such as complex conjugation when $\mathbb{K}$ is a totally complex extension of $\mathbb{Q}$. Consequently, the claim on the nature of $\mathcal{H}$ is clear.

Remark 5.4. The systems covered by Theorem 5.3 show many similarities with the $k$-free lattice points discussed earlier. In particular, they have positive topological entropy, which can in principle be determined from their description as weak model sets of maximal density in the sense of [6]. The spectral properties will reflect the comments made in Remark 4.2. We leave the details to the interested reader.

Unlike the situation in Theorem 4.5 , the group $\mathcal{H}$ will generally not be $\operatorname{Aut}(\Gamma) \simeq$ $\operatorname{GL}(d, \mathbb{Z})$, as we shall see in $\S 6$ below. In particular, for $M \in \operatorname{Aut}(\Gamma)$ and $\mathfrak{b} \in \mathcal{B}$, it need not be true that $M(\mathfrak{b})=\mathfrak{b}$ or $M\left(V_{\mathcal{B}}\right)=V_{\mathcal{B}}$. The following negative result, obtained via methods from analytic number theory, was pointed out to us by Blomer [11].

FACT 5.5. Let $M \in \mathrm{GL}(2, \mathbb{Z}) \backslash \mathrm{O}(2, \mathbb{Z})$. Then there exist Gaussian primes $\rho \in \mathbb{Z}[\mathrm{i}] \simeq \mathbb{Z}^{2}$ such that a positive proportion of square-free Gaussian integers $\alpha \in \mathbb{Z}[\mathrm{i}]$ satisfies $\rho^{2} \mid M \alpha$, and $M \alpha$ is thus not square-free in $\mathbb{Z}[\mathrm{i}]$. 
As we shall see in the next section, a simpler statement of purely algebraic nature exists, which suffices for our purposes and permits various generalizations.

\section{Power-free Gaussian and Eisenstein integers}

From now on, we shall need some classic results on quadratic number fields, which can all be drawn from [22, Chs. 14 and 15] or from [35]. To keep things simple, we only consider rings of integers that are Euclidean, so that we can easily work with primes and prime factorization (up to units) rather than with ideals; see [3] for various generalizations in our context.

As an example of an algebraic $\mathcal{B}$-free system that is Erdôs, let us view $\mathbb{Z}^{2}$ as $\mathbb{Z}[\mathrm{i}]$, the ring of Gaussian integers, and consider, for some fixed $2 \leqslant k \in \mathbb{N}$, the subset of $k$-free elements (to be defined below). $\mathbb{Z}[\mathrm{i}]$ is the maximal order in the quadratic field $\mathbb{Q}(\mathrm{i})$, and is Euclidean. The unit group of $\mathbb{Z}[\mathrm{i}]$ is

$$
\mathbb{Z}[\mathrm{i}]^{\times}=\{1, \mathrm{i},-1,-\mathrm{i}\} \simeq C_{4} .
$$

If $\mathcal{P}$ denotes the set of rational primes as before, the Gaussian primes [22, Theorem 252] can be represented by

$$
\mathcal{P}_{\mathrm{G}}=\{1+\mathrm{i}\} \cup\{p \in \mathcal{P}: p \equiv 3 \bmod 4\} \cup\{\pi, \bar{\pi}: \pi \bar{\pi}=p \in \mathcal{P} \text { with } p \equiv 1 \bmod 4\},
$$

where $\bar{r}$ is complex conjugation. The three subsets correspond to the ramified prime, where $(1+\mathrm{i})^{2}=2 \mathrm{i}$, the inert primes, and the (complex) splitting primes, respectively. Within the last, by a slight abuse of notation, we assume one representative pair for each $p$ to be selected, for instance by requiring $\pi$ to lie in the positive quadrant. This way, the representation of the primes is unique, and prime factorization works up to units.

Now, for any integer $k \geqslant 2$, we can define $V_{\mathrm{G}}^{(k)}$ as the set of Gaussian integers that are not divisible by the $k$ th power of any Gaussian prime. This is the set of $k$-free Gaussian integers. Figure 1 contains an illustration of the set $V_{\mathrm{G}}^{(2)}$, which was also used in [14]. We begin with a geometric symmetry consideration of $V_{\mathrm{G}}^{(k)}$ as follows.

LEMMA 6.1. Let $k \geqslant 2$ be fixed and let $A: \mathbb{Z}[\mathrm{i}] \longrightarrow \mathbb{Z}[\mathrm{i}]$ be a $\mathbb{Z}$-linear bijection that maps $V=V_{\mathrm{G}}^{(k)}$ into itself, $A(V) \subseteq V$. Then $A$ is a bijection of $U=\mathbb{Z}[\mathrm{i}]^{\times}$, and of $V$ as well. As such, it is of the form $A(x)=\epsilon \sigma(x)$ with $\epsilon \in U$ and $\sigma \in\left\{\mathrm{id},-{ }^{-}\right\}$, that is, $\epsilon$ is a unit and $\sigma$ a field automorphism of $\mathbb{Q}(\mathrm{i})$.

Together, these mappings form a group, which is the stabilizer of $\operatorname{Vin} \mathrm{GL}(2, \mathbb{Z})$, denoted by $\operatorname{stab}(V)$. The latter, for any $k \geqslant 2$, is the dihedral group $D_{4} \simeq C_{4} \rtimes C_{2}$ of order 8 , which is the symmetry group of the square and as such a maximal finite subgroup of $\mathrm{GL}(2, \mathbb{Z})$.

Proof. Clearly, any $A$ of the form $A(x)=\epsilon \sigma(x)$ maps units to units, and $V$ onto itself. Conversely, if $A$ preserves $U$ and $A(1)=\epsilon$, bijectivity of $A$ implies $A(\mathrm{i})=\mathrm{i} \epsilon$ or $A(\mathrm{i})=-\mathrm{i} \epsilon$, and $\mathbb{Z}$-linearity of $A$ determines the image of any $x \in \mathbb{Z}[\mathrm{i}]$ from here. This gives $A(x)=\epsilon x$ in the first case, and $A(x)=\epsilon \bar{x}$ in the second. It thus remains to show that any $\mathbb{Z}$-linear bijection $A$ of $\mathbb{Z}[\mathrm{i}]$ with $A(V) \subseteq V$ must preserve units. 
Let us begin with a simple but powerful observation on the coprimality structure of the $k$-free Gaussian integers. Consider $x \in V$, with $\operatorname{gcd}_{\mathrm{G}}(x, p)=1$ for every odd rational prime, where the $\operatorname{gcd}_{\mathrm{G}}$ in $\mathbb{Z}[\mathrm{i}]$ is unique up to units. Then $p^{\ell} x \in V$ for any $1 \leqslant \ell<k$, hence also $A\left(p^{k-1} x\right)=p^{k-1} A(x) \in V$, which implies $\operatorname{gcd}_{\mathrm{G}}(A(x), p)=1$. This argument cannot be extended to $p=2=-\mathrm{i}(1+\mathrm{i})^{2}$, which is ramified. Nevertheless, we may conclude that

$$
A(U) \subseteq U \cup(1+\mathrm{i}) U \cup \cdots \cup(1+\mathrm{i})^{k-1} U,
$$

where we now need to exclude all but the first set on the right-hand side.

Observe that, when $A$ is a mapping as specified, then so is the mapping $A^{\prime}$ defined by $A^{\prime}(x)=\epsilon A(x)$, for any $\epsilon \in U$. We may thus assume $A(1)=(1+\mathrm{i})^{m}$ for some integer $0 \leqslant m \leqslant k-1$ without loss of generality, matched by $A(\mathrm{i})=\kappa(1+\mathrm{i})^{n}$ with $\kappa \in U$ and $0 \leqslant n \leqslant k-1$. Now, from $\mathbb{Z}$-linearity in conjunction with bijectivity on $\mathbb{Z}[\mathrm{i}]$, we know that $\operatorname{det}(A)= \pm 1$, where

$$
\operatorname{det}(A)=\operatorname{Im}(\overline{A(1)} A(\mathrm{i}))=\operatorname{Im}\left(\kappa(1-\mathrm{i})^{m}(1+\mathrm{i})^{n}\right) .
$$

When $n \geqslant m$, this gives $\operatorname{det}(A)=2^{m} \operatorname{Im}\left(\kappa(1+\mathrm{i})^{n-m}\right)$, which cannot be unimodular unless $m=0$, so $A(1)=1$ and $\operatorname{det}(A)=\operatorname{Im}\left(\kappa(1+\mathrm{i})^{n}\right)$.

Observing $(1+\mathrm{i})^{2}=2 \mathrm{i}$, an analogous argument now also excludes $n \geqslant 2$, so $A(\mathrm{i})=\kappa$ or $A(\mathrm{i})=\kappa(1+\mathrm{i})$. In the first case, we get $A(\mathrm{i})=\mathrm{i}$ or $A(\mathrm{i})=-\mathrm{i}$ from bijectivity, and $A$ is also a bijection on $U$. When $A(\mathrm{i})=\kappa(1+\mathrm{i})$, we get $A(1 \pm \mathrm{i})=A(1) \pm A(\mathrm{i})=1 \pm \kappa(1+\mathrm{i})$. Irrespective of which unit $\kappa$ is, one of the images is an element of norm 5, where the norm refers to the field norm $\dagger$ of $x \in \mathbb{Q}(\mathrm{i})$, which is defined by $N(x)=x \bar{x}$ as usual. But such a norm value is impossible by our previous coprimality argument, and thus rules out this case.

When $m>n$, a completely analogous chain of arguments gives $n=0$ and $m=1$, which is then once again ruled out by the coprimality result. This leaves us with the mappings that preserve $U$ as claimed.

This result has the following immediate consequence, which can be seen as a simplified (and purely algebraic) case of Fact 5.5.

COROLLARY 6.2. Let $k \geqslant 2$ be a fixed integer and $V=V_{\mathrm{G}}^{(k)}$ the set of $k$-free Gaussian integers. If $A \in \mathrm{GL}(2, \mathbb{Z}) \backslash \operatorname{stab}(V)$, there exist a Gaussian prime $\rho$ and an element $w \in V$ such that $\rho^{k}$ divides $A(w)$.

No such prime can be inert, and it cannot be ramified when $k$ is even.

Proof. By Lemma 6.1, we know that $A \in \mathrm{GL}(2, \mathbb{Z})$ with $A(V) \subseteq V$ must be an element of $\operatorname{stab}(V)$, from which the first statement is clear.

The matrix $A$ is unimodular modulo $p^{k}$ for any rational prime $p$. As such, it cannot change the number of cosets of $p^{k} \mathbb{Z}^{2}$, and maps the zero coset onto itself. This rules out the case that $\rho \in \mathcal{P}_{\mathrm{G}}$ is inert.

$\dagger$ Note that the absolute norm of an ideal in $\mathbb{Z}[\mathrm{i}]$, which is always principal, agrees with the field norm of its generating element in this case. 
If $\rho=1+\mathrm{i}$, we have $N(\rho)=2$, and the same argument applies to $\rho$ when $k$ is even.

Under the identification of $\mathbb{Z}[\mathrm{i}]$ with $\mathbb{Z}^{2}$, let us now consider the subshifts

$$
\mathbb{X}_{\mathrm{G}}^{(k)}:=\overline{\mathbb{Z}^{2}+V_{\mathrm{G}}^{(k)}}
$$

which share many properties with our previous examples. In particular, they once again satisfy $\mathbb{X}_{\mathrm{G}}^{(k)}=\mathbb{A}$, with the appropriate notion for admissibility, and are hereditary. Further, they have pure point spectrum with trivial topological point spectrum, and the sets $V_{\mathrm{G}}^{(k)}$ are generic elements for the corresponding patch frequency (or Mirsky) measure, the latter defined via any averaging sequence of growing balls centred at 0 .

Proposition 6.3. Let $\left(\mathbb{X}_{\mathrm{G}}^{(k)}, \mathbb{Z}^{2}\right)$ with fixed $k \geqslant 2$ be the faithful shift generated by the $k$-free Gaussian integers. Then its centralizer is trivial, $\mathcal{S}=\mathbb{Z}^{2}$, while the normalizer $\mathcal{R}$ consists of affine transformations only. In particular, $\mathcal{R}$ contains a subgroup of the form $\mathbb{Z}^{2} \rtimes D_{4}$, where $D_{4}=\operatorname{stab}\left(V_{\mathrm{G}}^{(k)}\right)$ is the group from Lemma 6.1 .

Proof. The claim on the symmetries is a consequence of our general result in Theorem 5.3, which asserts that the centralizer is trivial, so $\mathcal{S}=\mathbb{Z}^{2}$.

For the extended symmetries, we are once more in the situation of diagram (5.1) from the proof of Theorem 5.3. Consequently, by Corollary 4.7, each element of the normalizer is an affine mapping, namely an element of the affine lattice group $\mathbb{Z}^{2} \rtimes \operatorname{GL}(2, \mathbb{Z})$.

That $\mathbb{Z}^{2} \rtimes D_{4}$ is a subgroup of $\mathcal{R}$ follows from Lemma 6.1. Indeed, since the $\mathbb{Z}^{2}$-orbit of $V_{\mathrm{G}}^{(k)}$ is dense in $\mathbb{X}_{\mathrm{G}}^{(k)}$ by construction and each element of $\mathcal{R}$ is continuous, any $M \in D_{4}$ maps $\mathbb{X}_{\mathrm{G}}^{(k)}$ onto itself, as does any affine mapping $(t, M)$ with $t \in \mathbb{Z}^{2}$ and $M \in D_{4}$.

It remains to complete the determination of $\mathcal{R}$, which leads to the following result.

THEOREM 6.4. The symmetry group and the extended symmetry group of $\left(\mathbb{X}_{\mathrm{G}}^{(k)}, \mathbb{Z}^{2}\right)$, with fixed $k \geqslant 2$, are given by $\mathcal{S}=\mathbb{Z}^{2}$ and $\mathcal{R}=\mathcal{S} \rtimes D_{4}$, respectively, where $D_{4}=\operatorname{stab}(V)=$ $C_{4} \rtimes C_{2}$ is the symmetry group of the square, and as such a maximal finite subgroup of $\mathrm{GL}(2, \mathbb{Z})$. In particular, $C_{4} \simeq \mathbb{Z}[\mathrm{i}]^{\times}$, while $C_{2}$ is the group of field automorphisms of $\mathbb{Q}(\mathrm{i})$, generated by complex conjugation.

Proof. The role of $\mathbb{Z}^{2} \rtimes D_{4}$ is clear from Proposition 6.3. To complete the proof, we need to show that the only $\mathbb{Z}$-linear, bijective mappings of $\mathbb{X}_{\mathrm{G}}^{(k)}$ onto itself are the ones we already know from Lemma 6.1.

As in the case of $k$-free lattice points, now by Theorem 5.3 , we have $\mathbb{X}_{\mathrm{G}}^{(k)}=\mathbb{A}$, where $\mathbb{A}$ is the subshift that consists of all admissible subsets of $V=V_{\mathrm{G}}^{(k)}$. Here, $V$ itself has the property that, for any $\pi \in \mathcal{P}_{\mathrm{G}}$, precisely the zero coset of the principal ideal $\left(\pi^{k}\right)$ is missing.

To complete the proof, we have to show that no $\mathbb{Z}$-linear bijection of $\mathbb{Z}[\mathrm{i}] \simeq \mathbb{Z}^{2}$ outside of $\operatorname{stab}(V)$ can map $\mathbb{A}$ into itself. So, let $A \in \mathrm{GL}(2, \mathbb{Z}) \backslash \operatorname{stab}(V)$. Then, by Corollary 6.2, there are a $\rho \in \mathcal{P}_{\mathrm{G}}$ and an element $w \in V$ such that $\rho^{k} \mid A(w)$. Set $n=N(\rho)^{k}$ and $z_{1}=w$. 
We will now choose Gaussian integers $z_{2}, \ldots, z_{n}$ such that the set $S=\left\{z_{1}, z_{2}, \ldots, z_{n}\right\}$ is admissible while $A(S)$ meets all cosets of the principal ideal $\left(\rho^{k}\right)$ in $\mathbb{Z}[\mathrm{i}]$.

To this end, choose a non-empty, finite set $P$ of Gaussian primes that contains all primes with $N(\pi)<N(\rho)$ but none with $N(\pi)=N(\rho)$. Concretely, when $N(\rho)>2$, we just take all primes of smaller norm, while we simply choose the inert prime 3 when $\rho=1+\mathrm{i}$. In any case, we have $P=\left\{\pi_{1}, \ldots, \pi_{m}\right\}$ with $m \geqslant 1$ this way.

Let $\mathcal{L}=\left(\pi_{1}^{k} \cdots \pi_{m}^{k}\right)$, which is a sublattice of $\mathbb{Z}[\mathrm{i}]$ of index $N\left(\pi_{1} \cdots \pi_{m}\right)^{k}$. Since this index is coprime with $n=N(\rho)^{k}$, we know from Fact 2.1 that $\mathcal{L}$ meets all cosets of $A^{-1}\left(\rho^{k}\right)$, and so does $1+\mathcal{L}$, as this is just a translate. Select numbers $z_{2}, \ldots, z_{n} \in 1+\mathcal{L}$ such that $A\left(z_{2}\right), \ldots, A\left(z_{n}\right)$ meet all non-zero cosets of $\left(\rho^{k}\right)$, and set $S:=\left\{z_{1}, \ldots, z_{n}\right\}$, with $z_{1}=w$. Clearly, the set $A(S)$ now meets all cosets of $\left(\rho^{k}\right)$ and is thus not admissible for $\rho$, so $A(S) \notin \mathbb{A}$. If we can show that $S$ itself is admissible for all Gaussian primes, we are done.

Clearly, $S$ is admissible for all Gaussian primes $\pi$ with $N(\pi)>N(\rho)$ by cardinality. If $S$ meets all cosets of $\left(\rho^{k}\right)$, each of them must occur precisely once. Then we modify $S$ via replacing $z_{2}$ by $z_{2}^{\prime}=z_{2}+w$, which reduces the number of cosets in $S$ by one, without reducing the number of cosets in $A(S)$ because $w$ is $k$-free with $A(w) \equiv 0 \bmod \left(\rho^{k}\right)$.

If $\rho$ is a splitting prime, we also have to check $\bar{\rho}$, which is not an associate but has the same norm. If $S$ meets all cosets of $\left(\bar{\rho}^{k}\right)$, we need to modify one element $z_{i}$ with $i>1$ to remove one coset from $S$. Due to the previous step, we can use neither $z_{2}^{\prime}$ nor the other element of $S$ that is now congruent to $z_{2}^{\prime}$ modulo $\left(\rho^{k}\right)$. Since $n \geqslant 4$, there is at least one other element, $z_{4}$ say, that can be replaced by $z_{4}+w$. The new set $S$ is now admissible for all Gaussian primes of norm at least $N(\rho)$, while $A(S)$ still meets all cosets of $\left(\rho^{k}\right)$ and is thus not in $\mathbb{A}$.

If $\rho \neq 1+\mathrm{i}$, it remains to see whether $S$ is now also admissible for all $\pi$ with $N(\pi)<N(\rho)$. By our construction with the lattice $\mathcal{L}$, we know that, modulo $\left(\pi^{k}\right)$, all $z_{i}$ are congruent to $w, 1$ or $1+w$, so we meet at most 3 cosets. Since $N(\pi)^{k} \geqslant 2^{k} \geqslant 4$, we are good, and $S$ is admissible for all Gaussian primes, while $A(S)$ is not, and we have the desired contradiction.

A completely analogous chain of arguments works for the ring of Eisenstein integers, $\mathbb{Z}[\rho]$, where $\rho=\mathrm{e}^{2 \pi \mathrm{i} / 3}=\frac{1}{2}(-1+\mathrm{i} \sqrt{3})$ is a primitive third root of unity. This is the ring of integers in the imaginary quadratic field $\mathbb{Q}(\rho)$, and is again Euclidean. The unit group is

$$
\mathbb{Z}[\rho]^{\times}=\left\{(-\rho)^{m}: 0 \leqslant m \leqslant 5\right\} \simeq C_{6},
$$

while the Eisenstein primes [22, Theorem 255], up to units, are represented by

$$
\mathcal{P}_{\mathrm{E}}=\{1-\rho\} \cup\{p \in \mathcal{P}: p \equiv 2 \bmod 3\} \cup\{\pi, \bar{\pi}: \pi \bar{\pi}=p \in \mathcal{P} \text { with } p \equiv 1 \bmod 3\},
$$

again in the order of the ramified prime, where $(1-\rho)^{2}=-3 \rho$, the inert primes, and the complex splitting primes, where one pair $(\pi, \bar{\pi})$ is selected for each $p$ in the last set.

Defining $V_{\mathrm{E}}^{(k)}$ for fixed $k \geqslant 2$ as the set of $k$-free Eisenstein integers, which we may view either as a subset of the triangular lattice, which is $\mathbb{Z}[\rho]$, or (equivalently) as one of the square lattice via $\left\{(m, n) \in \mathbb{Z}^{2}: m+n \rho \in V_{\mathrm{E}}^{(k)}\right\}$, the analogue of Lemma 6.1 now 
gives mappings of the form $A(x)=\epsilon \sigma(x)$ with $\epsilon \in \mathbb{Z}[\rho]^{\times}$and $\sigma \in\left\{\mathrm{id},{ }^{-}\right\}$, hence the group $D_{6} \simeq C_{6} \rtimes C_{2}$, which is another maximal finite subgroup of $\operatorname{GL}(2, \mathbb{Z})$, this time the one that is the symmetry group of the regular hexagon.

Defining the subshifts

$$
\mathbb{X}_{\mathrm{E}}^{(k)}:=\overline{\mathbb{Z}[\rho]+V_{\mathrm{E}}^{(k)}},
$$

one obtains the following analogue of Theorem 6.4, the proof of which need not be repeated, as the method is the same.

THEOREM 6.5. The symmetry group and the extended symmetry group of $\left(\mathbb{X}_{\mathrm{E}}^{(k)}, \mathbb{Z}[\rho]\right)$, with fixed $k \geqslant 2$, are given by $\mathcal{S}=\mathbb{Z}[\rho] \simeq \mathbb{Z}^{2}$ and $\mathcal{R}=\mathcal{S} \rtimes D_{6}$, respectively, where $D_{6}=C_{6} \rtimes C_{2}$ is the symmetry group of the regular hexagon, and as such isomorphic to a maximal finite subgroup of $\operatorname{GL}(2, \mathbb{Z})$. In particular, $C_{6}=\mathbb{Z}[\rho]^{\times}$, while $C_{2}$ is the group of field automorphisms of $\mathbb{Q}(\rho)$, generated by complex conjugation.

So far, we have seen extension groups that are either all of $\operatorname{GL}(2, \mathbb{Z})$ (for the visible lattice points), or finite subgroups thereof (for the $k$-free Gaussian or Eisenstein integers). In particular, the subshifts defined by the two examples illustrated in Figure 1 are clearly distinguished by different extended symmetry groups. At this point, it is a natural question whether infinite true subgroups of $\operatorname{GL}(2, \mathbb{Z})$ may also occur. To this end, we take a look at the corresponding dynamical systems for real quadratic fields.

\section{Power-free integers in real quadratic number fields}

Let us first consider subsets of $\mathbb{Z}^{2}$ constructed by means of $k$-free integers in $\mathbb{Z}[\sqrt{2}]$, namely

$$
V_{2}^{(k)}:=\left\{(m, n) \in \mathbb{Z}^{2}: m+n \sqrt{2} \text { is } k \text {-free in } \mathbb{Z}[\sqrt{2}]\right\},
$$

where $k \in \mathbb{N}$ with $k \geqslant 2$ is fixed. This set emerges via the isomorphism between $\mathbb{Z}^{2}$ and the Minkowski embedding of $\mathbb{Z}[\sqrt{2}]$ into $\mathbb{R}^{2}$ (cf. [4, §3.4.1]). Here, with $\lambda:=1+\sqrt{2}$ denoting the fundamental unit, the unit group is

$$
U=\mathbb{Z}[\sqrt{2}]^{\times}=\left\{ \pm \lambda^{n}: n \in \mathbb{Z}\right\} \simeq C_{2} \times C_{\infty},
$$

where we also note that $\mathbb{Z}[\sqrt{2}]=\mathbb{Z}[\lambda]$. This ring is again Euclidean, so we can work with unique prime decomposition up to units.

The primes [22, Theorem 256] can be represented as

$$
\mathcal{P}_{2}=\{\sqrt{2}\} \cup\{p \in \mathcal{P}: p \equiv \pm 3 \bmod 8\} \cup\left\{\pi, \pi^{\star}: \pi \pi^{\star}=p \in \mathcal{P} \text { with } p \equiv \pm 1 \bmod 8\right\},
$$

where $(\cdot)^{\star}$ denotes the mapping that is the unique extension of $\sqrt{2} \mapsto-\sqrt{2}$ to a field automorphism of the quadratic field $\mathbb{K}=\mathbb{Q}(\sqrt{2})$. The relevant field norm is then given by $N(x)=x x^{\star}$, which means $N(m+n \sqrt{2})=m^{2}-2 n^{2}$ or, equivalently, $N(r+s \lambda)=$ $r^{2}+2 r s-s^{2}$. Once again, to gain a representation modulo units (integers of norm \pm 1 ), one pair is selected in the last set for each $p$. Note that the field norm can be negative here, as a result of which the absolute norm of a principal ideal is now the absolute value of the field norm of a generating element. 
For some of the calculations below, it is helpful to express $\lambda^{n}$ in terms of $\lambda$ and 1 , for arbitrary $n \in \mathbb{Z}$. Defining the bi-infinite sequence $\left(c_{n}\right)_{n \in \mathbb{Z}}$ by the recursion $c_{n+1}=$ $2 c_{n}+c_{n-1}$ with initial conditions $c_{0}=0$ and $c_{1}=1$, one obtains the analogue of the Fibonacci numbers for the quadratic field $\mathbb{K}$. In particular, they satisfy $c_{-n}=(-1)^{n+1} c_{n}$ for all $n \in \mathbb{Z}$, and the first few numbers are

$$
\ldots, 29,-12,5,-2,1,0,1,2,5,12,29, \ldots
$$

The required formula for the units now reads

$$
\lambda^{n}=c_{n} \lambda+c_{n-1}=c_{n} \sqrt{2}+\left(c_{n}+c_{n-1}\right)
$$

which holds for all $n \in \mathbb{Z}$, as can easily be checked by induction.

LEMMA 7.1. Let $A: \mathbb{Z}[\sqrt{2}] \longrightarrow \mathbb{Z}[\sqrt{2}]$ be a $\mathbb{Z}$-linear bijection that maps $V=V_{2}^{(k)}$ into itself, for some fixed integer $k \geqslant 2$. Then $A$ is of the form $A(x)=\epsilon \sigma(x)$ with $\epsilon \in U$ and $\sigma \in\left\{\mathrm{id},(\cdot)^{\star}\right\}$, so maps $U=\mathbb{Z}[\sqrt{2}]^{\times}$onto itself. Together, these mappings form the group $\operatorname{stab}(V)=U \rtimes C_{2}=C_{2} \times\left(C_{\infty} \rtimes C_{2}\right)=C_{2} \times D_{\infty}$ of infinite order.

Proof. Any $A$ of the form $A(x)=\epsilon \sigma(x)$ satisfies $A(V)=V$ and maps $U$ onto itself, while the converse direction will be a consequence of showing that no further $\mathbb{Z}$-linear bijection of $\mathbb{Z}[\sqrt{2}]$ exists that maps the set $V$ into itself.

So, let $A$ be a $\mathbb{Z}$-linear bijection of $\mathbb{Z}[\sqrt{2}]$ with $A(V) \subseteq V$. As in the proof of Lemma 6.1, we observe that $x \in V$ with $\operatorname{gcd}_{\mathbb{K}}(x, p)=1$ for any odd $p \in \mathcal{P}$ implies $p^{k-1} x \in V$ and $A\left(p^{k-1} x\right)=p^{k-1} A(x) \in V$, hence $\operatorname{gcd}_{\mathbb{K}}(A(x), p)=1$ as well. Since $2=(\sqrt{2})^{2}$, which is the only ramified prime in this case, we see that $A(1)$ and $A(\sqrt{2})$ must be elements of the union

$$
U \cup \sqrt{2} U \cup 2 U \cup \cdots \cup(\sqrt{2})^{k-1} U
$$

where we may assume that we have, once again without loss of generality, $A(1)=2^{m / 2}$ and $A(\sqrt{2})=\kappa 2^{n / 2}$ with $\kappa \in U$ and $0 \leqslant m, n \leqslant k-1$. Here, we also know that this must result in a mapping with determinant \pm 1 .

Now, define $W: \mathbb{Q}(\sqrt{2}) \longrightarrow \mathbb{Q}$ by $W(x)=\left(x-x^{\star}\right) / 2 \sqrt{2}$, and observe that this gives $\operatorname{det}(A)=W\left(A(1)^{\star} A(\sqrt{2})\right)$, hence

$$
\operatorname{det}(A)=W\left(\kappa(-1)^{m}(\sqrt{2})^{m+n}\right) .
$$

When $m+n$ is even, so $m+n=2 \ell$, this means $\operatorname{det}(A)=(-1)^{m} 2^{\ell} W(\kappa)$, which can only be unimodular if $\ell=0$ and thus $m=n=0$. With $\kappa= \pm \lambda^{r}= \pm\left(c_{r} \sqrt{2}+\left(c_{r}+c_{r-1}\right)\right)$ from (7.1), we then get $\operatorname{det}(A)= \pm c_{r}$, which in turn implies $c_{r}=1$ and thus $r= \pm 1$. So, we have to consider $A(1)=1$ together with $A(\sqrt{2})= \pm \lambda$. Both choices, however, lead to a contradiction to our coprimality condition by observing that $2 \pm \sqrt{2}$, which has norm 2 , is then mapped under $A$ to $3+\sqrt{2}$, which is a number of norm 7 .

Likewise, when $m+n=2 \ell+1$, we have $\operatorname{det}(A)=(-1)^{m} 2^{\ell} W(\kappa \sqrt{2})$, which forces $\ell=0$ and thus either $m=1$ and $n=0$ or $m=0$ and $n=1$. In both cases, $\kappa= \pm \lambda^{r}$ can only lead to a unimodular determinant when $c_{r}+c_{r-1} \in\{ \pm 1\}$, which then 
means $r \in\{-1,0,1\}$. When $m=1$ and $n=0$, we get $A(1)=\sqrt{2}$ together with $A(\sqrt{2})=\kappa \in\left\{ \pm \lambda, \pm 1, \pm \lambda^{\star}\right\}$. All six choices lead to contradictions to coprimality with odd primes, by considering images of $1 \pm \sqrt{2}$ or $2 \pm \sqrt{2}$ under $A$.

It remains to consider $m=0$ and $n=1$, so $A(1)=1$ together with $A(\sqrt{2})=\kappa \sqrt{2}$, with the same options for $\kappa$ as in the previous case. Once again, $\kappa= \pm \lambda$ and $\kappa= \pm \lambda^{\star}$ are impossible, as can be seen by considering $A(1 \pm \sqrt{2})$. The choices $\kappa= \pm 1$, however, give the mappings $A(x)=x$ and $A(x)=x^{\star}$, which map $U$ onto itself, as does any multiplication of such an $A$ with an arbitrary $\epsilon \in U$.

Let us now consider the subshifts $\mathbb{X}_{2}^{(k)}:=\overline{\mathbb{Z}^{2}+V_{2}^{(k)}}$, in complete analogy to above.

PROPOSITION 7.2. The symmetry group and the extended symmetry group of $\left(\mathbb{X}_{2}^{(k)}, \mathbb{Z}^{2}\right)$, with fixed $k \geqslant 2$, are given by $\mathcal{S}=\mathbb{Z}^{2}$ and $\mathcal{R}=\mathcal{S} \rtimes \mathcal{H}$, respectively, where the extension group is $\mathcal{H}=\operatorname{stab}\left(V_{2}^{(k)}\right)=U \rtimes C_{2} \simeq C_{2} \times D_{\infty}$, which is infinite.

Proof. From Lemma 7.1, we see that $\mathbb{Z}^{2} \rtimes \mathcal{H}$ is a subgroup of $\mathcal{R}$. The latter is a subgroup of $\mathbb{Z}^{2} \rtimes \mathrm{GL}(2, \mathbb{Z})$ by Corollary 4.7 . It thus remains to show that $\mathcal{H}=\operatorname{stab}\left(V_{2}^{(k)}\right)$ contains all $\operatorname{GL}(2, \mathbb{Z})$ elements that map $\mathbb{X}_{2}^{(k)}$ into itself. This last step can be established by the method from the proof of Theorem 6.4, with the field norm replaced by the absolute norm.

There are other real quadratic fields that are Euclidean, such as $\mathbb{Q}(\sqrt{m})$ with $m=5$ and $m=3$, which play prominent roles in the theory of aperiodic order, as they are connected with systems with fivefold and 12-fold symmetry, respectively; see [4, §2.5.1] for background.

For $m=5$, the ring of integers is $\mathbb{Z}[\tau]$, where $\tau=\frac{1}{2}(1+\sqrt{5})$ is the golden ratio. This ring has unit group $U=\mathbb{Z}[\tau]^{\times}=\left\{ \pm \tau^{n}: n \in \mathbb{Z}\right\}$, and the primes [22, Theorem 257] are represented by

$$
\mathcal{P}_{5}=\{\sqrt{5}\} \cup\{p \in \mathcal{P}: p \equiv \pm 2 \bmod 5\} \cup\left\{\pi, \pi^{\star}: \pi \pi^{\star}=p \in \mathcal{P} \text { with } p \equiv \pm 1 \bmod 5\right\},
$$

where $(\cdot)^{\star}$ is the field automorphism of $\mathbb{Q}(\sqrt{5})$ induced by $\sqrt{5} \mapsto-\sqrt{5}$, with our usual convention for the splitting primes in place. The only ramified prime is 5 , while the field norm on $\mathbb{Z}[\tau]$ is $N(m+n \tau)=m^{2}+m n-n^{2}$, which can be negative.

Finally, let us consider the slightly more complicated case $m=3$, where the ring of integers is $\mathbb{Z}[\sqrt{3}]$. Its unit group is given by $\mathbb{Z}[\sqrt{3}]^{\times}=\left\{ \pm \eta^{n}: n \in \mathbb{Z}\right\}$, with fundamental unit $\eta=2+\sqrt{3}$. Here, in contrast to the two previous cases, all units have norm 1 . Employing [35, Theorem 11.1], one sees that the primes up to units can be represented as

$$
\begin{aligned}
\mathcal{P}_{3}= & \{1+\sqrt{3}, \sqrt{3}\} \cup\{p \in \mathcal{P}: p \equiv \pm 5 \bmod 12\} \\
& \cup\left\{\pi, \pi^{\star}: \pi \pi^{\star}= \pm p \in \mathcal{P} \text { with } p \equiv \pm 1 \bmod 12\right\}
\end{aligned}
$$

with the usual convention for the last set, where $(\cdot)^{\star}$ is now induced by $\sqrt{3} \mapsto-\sqrt{3}$. Unlike before, since the field discriminant is 12 and thus divisible by 2 and 3, there are two ramified primes, where $(1+\sqrt{3})^{2}=2 \eta$ is the additional relation. 
This leads to more cases to consider in the determination of $\operatorname{stab}\left(V_{3}^{(k)}\right)$, but the $\mathbb{Z}$-linear bijections of $\mathbb{Z}[\sqrt{3}]$ that map $V_{3}^{(k)}$ into itself, for some fixed $k \geqslant 2$, are still the expected ones, namely the maps $A$ of the form $A(x)=\epsilon \sigma(x)$ with $\epsilon \in U=\mathbb{Z}[\sqrt{3}]^{\times}$and $\sigma \in\left\{\mathrm{id},(\cdot)^{\star}\right\}$; we leave this proof to the interested reader.

In both cases, a proof analogous to the one of Proposition 7.2 gives the following result.

THEOREM 7.3. The symmetry group and the extended symmetry group of $\left(\mathbb{X}_{m}^{(k)}, \mathbb{Z}^{2}\right)$, with fixed $m \in\{2,3,5\}$ and $k \geqslant 2$, are given by $\mathcal{S}=\mathbb{Z}^{2}$ and $\mathcal{R}=\mathcal{S} \rtimes \mathcal{H}$, respectively, where the extension group is $\mathcal{H}=\operatorname{stab}\left(V_{m}^{(k)}\right)=U \rtimes C_{2} \simeq C_{2} \times D_{\infty}$, which is an infinite group that does not depend on $k$, where $U$ is the unit group.

The advantage of using the normalizer in addition to the centralizer as a topological invariant becomes obvious in dimensions $d \geqslant 2$. In [9], this was demonstrated for the chair tiling shift and for Ledrappier's shift. In both cases, $\mathcal{R}$ was an extension of $\mathcal{S}$ of finite index. As our number-theoretic examples above show, this phenomenon occurs again, but $\mathcal{R}$ can also be an infinite-index extension of $\mathcal{S}$, either for trivial reasons (visible lattice points) or for non-trivial ones ( $k$-free $\mathbb{Z}[\sqrt{2}]$-integers). At present, we do not know whether such an infinite extension is also possible for minimal, deterministic (zero-entropy) subshifts. In any case, these groups allow the distinction of several subshifts (up to topological conjugacy) that have the same centralizer, but different normalizers, such as $\left(\mathbb{X}_{V}, \mathbb{Z}^{2}\right)$ and $\left(\mathbb{X}_{\mathrm{G}}^{(2)}, \mathbb{Z}^{2}\right)$ from above.

Due to the nature of the associated dynamical system, the structure of the hull $\mathbb{X}_{V}$ (given the property of hereditariness and the natural topology employed) allows for local symmetries (that is, transformations that preserve finite local substructures in the set $V$ up to translation) to manifest themselves. To some extent, they may be observed by analysing the extended symmetry group $\mathcal{R}\left(\mathbb{X}_{V}\right)$. While symmetries of the set $V$ in the standard sense (global symmetries) are obviously local symmetries in this new sense, the converse is not clear. It is easy to build sets $V$ that have many local symmetries while lacking global symmetries entirely. Thus, it is interesting to note that, in the current context, those two kinds of symmetries happen to be the same, giving rise to the question whether this is a natural phenomenon on sets defined in an 'algebraic' form in more general ways.

This setting deserves further attention, in particular in the context of dynamical systems of number-theoretic origin. As this will require a more general approach via ideals, as well as some additional and less elementary results from algebraic and analytic number theory, we defer this to a separate investigation [3].

Acknowledgements. MB would like to thank Valentin Blomer for helpful discussions and hints. We thank the referee for a number of suggestions which helped to improve the presentation. Moreover, AB is grateful to ANID (formerly CONICYT) for the financial support received under the Doctoral Fellowship ANID-PFCHA/Doctorado Nacional/2017-21171061, ML was supported by Narodowe Centrum Nauki grant UMO-2019/33/B/ST1/00364, and AN acknowledges financial support by the German Research Foundation (DFG) through its Heisenberg program (project number 437113953). 


\section{REFERENCES}

[1] T. M. Apostol. Introduction to Analytic Number Theory, corr. 4th printing. Springer, New York, 1984.

[2] M. Baake. A brief guide to reversing and extended symmetries of dynamical systems. Ergodic Theory and Dynamical Systems in their Interactions with Arithmetics and Combinatorics (Lecture Notes in Mathematics, 2213). Ed. S. Ferenczi, J. Kułaga-Przymus and M. Lemańczyk. Springer, Cham, 2018, pp. 117-135.

[3] M. Baake, Á. Bustos and A. Nickel. On the stabiliser of some number-theoretic shift spaces, in preparation.

[4] M. Baake and U. Grimm. Aperiodic Order. Vol. 1. A Mathematical Invitation. Cambridge University Press, Cambridge, 2013.

[5] M. Bake and C. Huck. Ergodic properties of visible lattice points. Proc. Steklov Inst. Math. 288 (2015), 184-208.

[6] M. Baake, C. Huck and N. Strungaru. On weak model sets of extremal density. Indag. Math. 28 (2017), 3-31.

[7] M. Baake and D. Lenz. Dynamical systems on translation bounded measure: pure point dynamical and diffraction spectra. Ergodic Th. \& Dynam. Sys. 24 (2004), 1867-1893.

[8] M. Baake, R. V. Moody and P. A. B. Pleasants. Diffraction of visible lattice points and $k$ th power free integers. Discrete Math. 221 (2000), 3-42.

[9] M. Baake, J. A. G. Roberts and R. Yassawi. Reversing and extended symmetries of shift spaces. Discrete Contin. Dyn. Syst. A 38 (2018), 835-866.

[10] M. Baake, T. Spindeler and N. Strungaru. Diffraction of compatible random substitutions in one dimension. Indag. Math. 29 (2018), 1031-1071

[11] V. Blomer. Private communication, 2017.

[12] Z. I. Borevich and I. R. Shafarevich. Number Theory. Academic Press, New York, 1966.

[13] Á. Bustos. Extended symmetry groups of multidimensional subshifts with hierarchical structure. Discrete Contin. Dyn. Syst. A 40 (2020), 5869-5895.

[14] F. Cellarosi and I. Vinogradov. Ergodic properties of $k$-free integers in number fields. J. Mod. Dyn. 7 (2013), 461-488.

[15] M. I. Cortez and S. Petite. Realization of big centralizers of minimal aperiodic actions on the Cantor set. Discrete Contin. Dyn. Syst. A 40 (2020), 2891-2901.

[16] E. M. Coven, A. Quas and R. Yassawi. Computing automorphism groups of shifts using atypical equivalence classes. Discrete Anal. 3 (2016), 1-24.

[17] V. Cyr and B. Kra. The automorphism group of a shift of linear growth: beyond transitivity. Forum Math. Sigma 3 (2015), e5:1-27.

[18] S. Donoso, F. Durand, A. Maass and S. Petite. On automorphism groups of low complexity subshifts. Ergodic Th. \& Dynam. Sys. 36 (2016), 64-95.

[19] A. Dymek, S. Kasjan, J. Kułaga-Przymus and M. Lemańczyk. $\mathcal{B}$-free sets and dynamics. Trans. Amer. Math. Soc. 370 (2018), 5425-5489.

[20] E. H. El Abdalaoui, M. Lemańczyk and T. de la Rue. A dynamical point of view on the set of $\mathcal{B}$-free integers. Int. Math. Res. Not. 16 (2015), 7258-7286.

[21] G. Goodson, A. del Junco, M. Lemańczyk and D. Rudolph. Ergodic transformations conjugate to their inverses by involutions. Ergodic Th. \& Dynam. Sys. 16 (1996), 97-124.

[22] G. M. Hardy and E. M. Wright. An Introduction to the Theory of Numbers, 6th edn. Ed. D. R. Heath-Brown and J .H. Silverman. Oxford University Press, Oxford, 2008.

[23] S. Kasjan, G. Keller and M. Lemańczyk. Dynamics of $\mathcal{B}$-free sets: a view through the window. Int. Math. Res. Not. 9 (2019), 2690-2734.

[24] G. Keller. Maximal equicontinuous generic factors and weak model sets. Discrete Contin. Dyn. Syst. A, 40 (2020), 6855-6875.

[25] G. Keller. Private communication, 2019.

[26] Y.-O. Kim, J. Lee and K. K. Park. A zeta function for flip systems. Pacific J. Math. 209 (2003), $289-301$.

[27] B. Kitchens and K. Schmidt. Isomorphism rigidity of irreducible algebraic $\mathbb{Z}^{d}$-actions. Invent. Math. 142 (2000), 559-577.

[28] D. Lind and B. Marcus. An Introduction to Symbolic Dynamics and Coding. Cambridge University Press, New York, 1995.

[29] M. K. Mentzen. Automorphisms of subshifts defined by $\mathcal{B}$-free sets of integers. Colloq. Math. 147 (2017), $87-94$.

[30] J. Neukirch. Algebraic Number Theory. Springer, Berlin, 1999.

[31] A. G. O'Farrel and I. Short. Reversibility in Dynamics and Group Theory. Cambridge University Press, Cambridge, 2015. 
[32] P. A. B. Pleasants and C. Huck. Entropy and diffraction of the $k$-free points in $n$-dimensional lattices. Discrete Comput. Geom. 50 (2013), 39-68.

[33] J. A. G. Roberts and G. R. W. Quispel. Chaos and time-reversal symmetry: order and chaos in reversible dynamical systems. Phys. Rep. 216 (1992), 63-177.

[34] K. Schmidt. Dynamical Systems of Algebraic Origin. Birkhäuser, Basel, 1995.

[35] D. Zagier. Zetafunktionen und quadratische Körper. Springer, Berlin, 1981. 